Pacific

Journal of

Mathematics

\title{
ON DEFORMATION QUANTIZATIONS OF HYPERTORIC VARIETIES
}

GWYN BELlamy AND TOSHIRO KUWABARA 


\title{
ON DEFORMATION QUANTIZATIONS OF HYPERTORIC VARIETIES
}

\author{
GWYN BELlamy AND TOSHIRO KuWABARA
}

\begin{abstract}
Based on a construction by Kashiwara and Rouquier, we present an analogue of the Beilinson-Bernstein localization theorem for hypertoric varieties. In this case, sheaves of differential operators are replaced by sheaves of $W$-algebras. As a special case, our result gives a localization theorem for rational Cherednik algebras associated to cyclic groups.
\end{abstract}

\section{Introduction}

Kontsevich [2001] and Polesello and Schapira [2004] have shown that one can construct a stack of " $W$-algebroids" (or deformation-quantization algebroids) on any symplectic manifold. These stacks of $W$-algebroids provide a quantization of the sheaf of holomorphic functions on the manifold. In certain cases, these stacks of $W$-algebroids are the algebroids associated to a sheaf of noncommutative algebras called $W$-algebras. Locally this is always the case. When the symplectic manifold in question is the Hamiltonian reduction of a space equipped with a genuine sheaf of $W$-algebras, Kashiwara and Rouquier [2008] have shown that one can define a family of sheaves of $W$-algebras on the Hamiltonian reduction coming from the sheaf upstairs. This provides a large class of examples of sheaves of $W$-algebras on nontrivial symplectic manifolds. In this paper we study $W$-algebras on the simplest class of Hamiltonian reductions, those coming from the action of a torus $T$ on a symplectic vector space $V$. These spaces $Y(A, \delta)$, where $A$ is a matrix encoding the action of $T$ on $V$ and $\delta \in \mathbb{X}(T)$ is a character of $T$, are called hypertoric varieties. They were originally studied as hyperkähler manifolds by Bielawski and Dancer [2000]. Examples of hypertoric varieties include the cotangent space of projective $n$-space and resolutions of cyclic Kleinian singularities. More generally,

Kuwabara was partially supported by Grant-in-Aid for Young Scientists (B) 21740013, and by GCOE "Fostering top leaders in mathematics", Kyoto University. He was also partially supported by Basic Science Research Program through the National Research Foundation of Korea (NRF) grant funded by the Korea government (MEST)(2010-0019516).

MSC2010: primary 14A22, 16S80; secondary 18E35.

Keywords: deformation quantization, hypertoric varieties. 
the cotangent space of any smooth toric variety can be realized as a dense, open subvariety of the corresponding hypertoric variety.

One can also associate to the data of a reductive group $G$ acting on a symplectic vector space a certain family of noncommutative algebras $U_{\chi}$, where $\chi \in \mathbb{X}(\mathfrak{g})$ is a character of $\mathfrak{g}=\operatorname{Lie}(G)$, called quantum Hamiltonian reductions. In the case $G=T$ is a torus, these algebras have been extensively studied by Musson and Van den Bergh [1998]. The main goal of this paper is to prove a localization theorem, analogous to the celebrated Beilinson-Bernstein localization theorem [1981], giving an equivalence between the category of finitely generated modules for the quantum Hamiltonian reduction and a certain category of modules for a $W$-algebra. When the character $\delta$ is chosen to lie in the interior $C$ of a G.I.T. chamber in $\mathbb{X}(T)$, the hypertoric variety $Y(A, \delta)$ is a symplectic manifold. Then each character $\chi \in \mathbb{X}(\mathfrak{t})$ gives a sheaf of $W$-algebras $\mathscr{A}_{\chi}$ on $Y(A, \delta)$. Associated to $\mathscr{A}_{\chi}$ is a category of "good" $\mathscr{A}_{\chi}$-modules, $\operatorname{Mod}_{F}^{\text {good }}\left(\mathscr{A}_{\chi}\right)$ and a subcategory $\operatorname{Mod}_{F}^{\text {good }}\left(\mathscr{A}_{\chi}\right)$ consisting (roughly) of those modules generated by their global section (the reader is referred to section 2 for the precise definition of these categories). Then we have natural localization and global section functors

$$
\begin{aligned}
& \text { Loc }: \mathrm{U}_{\chi}-\bmod \longrightarrow \operatorname{Mod}_{F}^{\text {good }}\left(\mathscr{A}_{\chi}\right), \quad \operatorname{Loc}(M)=\mathscr{A}_{\chi} \otimes \mathrm{U}_{\chi} M, \\
& \text { Sec }: \operatorname{Mod}_{F}^{\text {good }}\left(\mathscr{A}_{\chi}\right) \longrightarrow \mathrm{U}_{\chi}-\bmod , \quad \operatorname{Sec}(M)=\operatorname{Hom}_{\operatorname{Mod}_{F}^{\text {good }}\left(\mathscr{A}_{\chi}\right)}\left(\mathscr{A}_{\chi}, M\right) .
\end{aligned}
$$

Our main result can be stated as follows.

Theorem 1.1. Let $\chi \in C_{\mathbb{Q}}$.

(i) The functor Loc defines an equivalence of categories $\mathrm{U}_{\chi}$-mod $\stackrel{\sim}{\longrightarrow} \underline{\operatorname{Mod}}_{F}^{\text {good }}\left(\mathscr{A}_{\chi}\right)$ with quasiinverse Sec.

(ii) There exists some $\theta \in C \cap \mathbb{X}(T)$ such that the functor Loc defines an equivalence of categories $\mathrm{U}_{\chi+\theta}$-mod $\stackrel{\sim}{\longrightarrow} \operatorname{Mod}_{F}^{\text {good }}\left(\mathscr{A}_{\chi+\theta}\right)$ with quasiinverse Sec.

The theorem shows that localization always gives an equivalence of categories, provided one is sufficiently far away from the G.I.T. walls.

Corollary 1.2. Let $\chi \in C_{\mathbb{Q}}$. If the global dimension of $\mathrm{U}_{\chi}$ is finite then the functor Loc defines an equivalence of categories $\mathrm{U}_{\chi}$-mod $\stackrel{\sim}{\longrightarrow} \operatorname{Mod}_{F}^{\text {good }}\left(\mathscr{A}_{\chi}\right)$ with quasiinverse Sec.

A particular class of examples of hypertoric varieties are the minimal resolutions $\left(\mathbb{C}^{2} / \mathbb{Z}_{m}\right)^{\sim}$ of the Kleinian singularities of type $A$. Under mild restrictions on the parameters, the corresponding quantum Hamiltonian reductions are Morita equivalent to the rational Cherednik algebras $H_{\boldsymbol{h}}$ associated to cyclic groups. Then a corollary of our main result is a localization theorem for these rational Cherednik algebras. 
Corollary 1.3. For $\boldsymbol{h}$ not lying on a G.I.T. wall, the functor $\operatorname{Loc}(e \cdot(\cdot))$ defines an equivalence of categories

$$
H_{\boldsymbol{h}}-\bmod \stackrel{\sim}{\longrightarrow} \operatorname{Mod}_{F}^{\text {good }}\left(\mathscr{A}_{\boldsymbol{h}}\right)
$$

with quasiinverse $H_{h} e \otimes_{e H_{h} e} \operatorname{Sec}(\cdot)$.

We summarize the content of each section. In Section 2 we introduce, following Kashiwara and Rouquier, $W$-algebras on symplectic manifolds in the equivariant setting. In Section 3 we give a criterion for the $W$-affinity of a class of $W$-algebras on those symplectic manifolds that are obtained by Hamiltonian reduction of a vector space acted upon by a reductive group. The $W$-algebras on hypertoric varieties that we will consider later are a special case of this more general setup. The main result of this section is Theorem 3.3.

Hypertoric varieties are introduced in Section 4 and we show that they possess the correct geometric properties that are required to apply the results of Section 3. Using the results of Musson and Van den Bergh, we prove our main results, Theorem 5.2 and Corollary 5.3. In the final section we consider the special case where the hypertoric variety is the resolution of a Kleinian singularity of type $A$ and the global sections of the sheaf of $W$-algebras on this resolution can be identified with the spherical subalgebra of the rational Cherednik algebra associated to a cyclic group.

Convention. Throughout, a variety will always mean an integral, separated scheme of finite type over $\mathbb{C}$. A nonreduced space will be referred to as a scheme, again assumed to be over $\mathbb{C}$.

\section{2. $W$-algebras}

2A. In this section we recall the definition of $W$-algebras as given in [Kashiwara and Rouquier 2008]. We state results about the existence and "affinity" of $W$-algebras. Let $X$ be a complex analytic manifold and let $O_{X}$ denote the sheaf of regular, holomorphic functions on $X$. Denote by $\mathscr{D}_{X}$ the sheaf of differential operators on $X$ with holomorphic coefficients. Denote by $\boldsymbol{k}=\mathbb{C}((\hbar))$ the field of formal Laurent series in $\hbar$ and by $\boldsymbol{k}(0)$ the subring $\mathbb{C} \llbracket \hbar \rrbracket$ of formal functions on $\mathbb{C}$. Considering $\boldsymbol{k}$ and $\boldsymbol{k}(0)$ as abelian groups, the corresponding sheaves of locally constant functions on $X$ will be denoted $\boldsymbol{k}_{X}$ and $\boldsymbol{k}(0)_{X}$ respectively. Given $m \in \mathbb{Z}$, we define $\mathscr{W}_{T^{*} \mathbb{C}^{n}}(m)$ to be the sheaf of formal power series $\sum_{i \geq-m} \hbar^{i} a_{i}, a_{i} \in \mathbb{O}_{T^{*} \mathbb{C}^{n}}$, on the cotangent bundle $T^{*} \mathbb{C}^{n}$ of $\mathbb{C}^{n}$. Let us fix coordinates $x_{1}, \ldots, x_{n}$ on $\mathbb{C}^{n}$ and dual coordinates $\xi_{1}, \ldots, \xi_{n}$ on $\left(\mathbb{C}^{n}\right)^{*}$, identifying $T^{*} \mathbb{C}^{n}$ with $\mathbb{C}^{n} \times\left(\mathbb{C}^{n}\right)^{*}$. Set $\mathcal{W}_{T^{*} \mathbb{C}^{n}}=\bigcup_{m \in \mathbb{Z}} \mathcal{W}_{T^{*} \mathbb{C}^{n}}(m)$. Then $\mathcal{W}_{T^{*} \mathbb{C}^{n}}$ is a sheaf of (noncommutative) $\boldsymbol{k}$-algebras 
on $T^{*} \mathbb{C}^{n}$. Multiplication is defined by

$$
a \circ b=\sum_{\alpha \in \mathbb{Z}_{\geq 0}^{n}} \frac{\hbar^{|\alpha|}}{\alpha !} \partial_{\xi}^{\alpha} a \cdot \partial_{x}^{\alpha} b,
$$

where $|\alpha|=\sum_{i=1}^{n} \alpha_{i}, \alpha !=\alpha_{1} ! \cdots \alpha_{n} !$ and $\partial_{\xi}^{\alpha}=\partial^{|\alpha|} /\left(\partial^{\alpha_{1}} \xi_{1} \cdots \partial^{\alpha_{n}} \xi_{n}\right)$. There is a ring homomorphism $\mathscr{D}_{\mathbb{C}^{n}}\left(\mathbb{C}^{n}\right) \rightarrow \mathscr{W}_{T^{*} \mathbb{C}^{n}}\left(T^{*} \mathbb{C}^{n}\right)$ given by $x_{i} \mapsto x_{i}$ and $\partial / \partial x_{i} \mapsto \hbar^{-1} \xi_{i}$. Note that $\mathscr{W}_{T^{*} \mathbb{C}^{n}}(0)$ is a $\boldsymbol{k}(0)$-subalgebra. We denote the symbol map for $\mathscr{W}_{T^{*} \mathbb{C}^{n}}$ by

$$
\sigma_{m}: \mathscr{W}_{T^{*} \mathbb{C}^{n}}(m) \longrightarrow \mathcal{W}_{T^{*} \mathbb{C}^{n}}(m) / \mathscr{W}_{T^{*} \mathbb{C}^{n}}(m-1) \simeq \hbar^{-m} \mathbb{O}_{T^{*} \mathbb{C}^{n}}
$$

The sheaf $\mathbb{O}_{T^{*} \mathbb{C}^{n}}$ is a sheaf of Poisson algebras with Poisson bracket given by

$$
\left\{x_{i}, x_{j}\right\}=\left\{\xi_{i}, \xi_{j}\right\}=0, \quad\left\{\xi_{i}, x_{j}\right\}=\delta_{i j} \quad \text { for all } i, j \in[1, n] .
$$

One sees from (1) that $\sigma_{0}\left(\hbar^{-1}[a, b]\right)=\left\{\sigma_{0}(a), \sigma_{0}(b)\right\}$ for all $a, b \in \mathcal{W}_{T^{*} \mathbb{C}^{n}}(0)$.

2B. Let us now assume that $X$ is a complex symplectic manifold with holomorphic 2-form $\omega_{X}$. A map $f$ between open subsets $U \subset X$ and $V \subset Y$ of the symplectic manifolds $\left(X, \omega_{1}\right)$ and $\left(Y, \omega_{2}\right)$ is said to be a symplectic map if $f^{*} \omega_{2}=\omega_{1}$. A symplectic map is always locally biholomorphic [Björk 1979, Lemma 5.5.2], therefore by symplectic map we will actually mean a biholomorphic symplectic map. Based on [Kontsevich 2001; Polesello and Schapira 2004], we have:

Definition 2.1. A $W$-algebra on $X$ is a sheaf of $\boldsymbol{k}$-algebras $\mathcal{W}_{X}$ together with a $\boldsymbol{k}(0)$ subalgebra $\mathscr{W}_{X}(0)$ such that for each point $x \in X$ there exists an open neighborhood $U$ of $x$ in $X$, a symplectic map $f: U \longrightarrow V \subset T^{*} \mathbb{C}^{n}$ and a $\boldsymbol{k}$-algebra isomorphism $r:\left.f^{-1}\left(\left.\mathcal{W}_{T^{*} \mathbb{C}^{n}}\right|_{V}\right) \stackrel{\sim}{\longrightarrow} \mathcal{W}_{X}\right|_{U}$ such that:

(i) The isomorphism $r$ restricts to a $\boldsymbol{k}(0)$-isomorphism

$$
\left.f^{-1}\left(\left.\mathcal{W}_{T^{*} \mathbb{C}^{n}}(0)\right|_{V}\right) \stackrel{\sim}{\longrightarrow} \mathcal{W}_{X}(0)\right|_{U}
$$

(ii) Setting $\mathcal{W}_{X}(m)=\hbar^{-m} \mathcal{W}(0)$ for all $m \in \mathbb{Z}$, we have

$$
\sigma_{0}: W_{X}(0) \longrightarrow \mathcal{W}_{X}(0) / \mathcal{W}_{X}(-1) \simeq \mathcal{O}_{X}
$$

and the following diagram commutes:

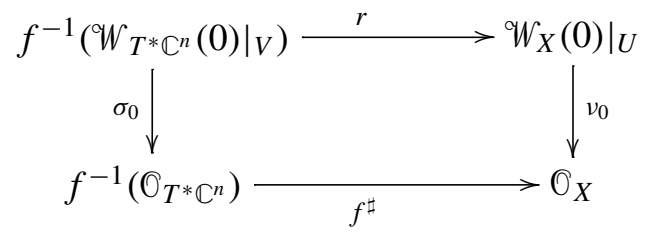


2C. The first statement of property (ii) of Definition 2.1 is actually a consequence of property (i). Next, Definition 2.1(ii) implies that $\sigma_{0}\left(\hbar^{-1}[a, b]\right)=\left\{\sigma_{0}(a), \sigma_{0}(b)\right\}$ for all $a, b \in \mathscr{W}_{X}(0)$, where the Poisson bracket on $\mathscr{O}_{X}$ is the one induced from the symplectic form $\omega$ on $X$.

2D. Categories of $\boldsymbol{W}$-modules. Unless explicitly stated, all modules will be left modules. Since $\mathscr{W}_{X}(0)$ is Noetherian (see [Kashiwara and Rouquier 2008, (2.2.2)]), a $W_{X}(0)$-module $\mathcal{M}$ is said to be coherent if it is locally finitely generated. For a $W_{X}$-module $\mathcal{M}$, a $\mathcal{W}_{X}(0)$-lattice of $\mathcal{M}$ is a $\mathcal{W}_{X}(0)$-submodule $\mathcal{N}$ of $\mathcal{M}$ such that the natural map $\mathcal{W} \otimes W(0) \mathcal{N} \rightarrow \mathcal{M}$ is an isomorphism. A $\mathcal{W}$-module $\mathcal{M}$ is said to be good if for every relatively compact open set $U$ there exists a coherent $\left.\mathscr{W}_{X}(0)\right|_{U}$-lattice for $\left.\mathcal{M}\right|_{U}$. We will denote the category of left $\mathscr{W}_{X}$-modules as $\operatorname{Mod}\left(\mathscr{W}_{X}\right)$ and the full subcategory of good $\mathcal{W}_{X}$-modules as $\operatorname{Mod}^{\text {good }}\left(W_{X}\right)$. It is an abelian subcategory. If $M(0)$ is a $W_{X}(0)$-lattice of $M$, set $M(m):=\hbar^{-m} \mathcal{M}(0)$.

Lemma 2.2. Let $\mathcal{M}$ be a coherent $\mathscr{W}_{X}$-module, equipped with a global $W_{X}(0)$ lattice $M(0)$. Then the filtration $M(n), n \in \mathbb{Z}$, is exhaustive, Hausdorff and complete; that is,

(i) $\bigcup_{n \in \mathbb{Z}} M(n)=M$,

(ii) $\bigcap_{n \in \mathbb{Z}} M(n)=0$,

(iii) $\lim _{-\infty \leftarrow n} M / M(n)=M$.

(Our terminology is chosen to agree with that of [Weibel 1994, §5].)

Proof. The statement (i) is true if $M=W_{X}$. But, by the definition of a lattice, we have

$$
\bigcup_{n \in \mathbb{Z}} \mathcal{M}(n)=\bigcup_{n \in \mathbb{N}} W_{X}(n) \otimes W_{X}(0) \mu(0)=W_{X} \otimes W_{X}(0) \mu(0)=\mu .
$$

Part (ii) follows from [Kashiwara and Rouquier 2008, Lemma 2.11]. Fix some open subset $U$ of $X$ and take a section $\left(f_{n}\right)_{n \in \mathbb{Z}} \in \lim _{-\infty \leftarrow n}(\mathcal{M} / M(n))(U)$. Then, by part (i), there exists some integer $k>n$ such that the image $f_{n}$ of $f$ in $(\mathcal{M} / \mathcal{M}(n))(U)$ lies in $(M(k) / M(n))(U)$. Now by definition $f_{n}$ is the image of $f_{n-1}$ in the surjection

$$
(M / M(n-1))(U) \longrightarrow(M / M(n))(U),
$$

hence $f_{n-1} \in(M(k) / M(n-1))(U)$ too. Thus $\left(\hbar^{-k} f_{n}\right)_{n \in \mathbb{Z}}$ is in

$$
\lim _{-\infty \leftarrow n}(\mathcal{M}(0) / \mathcal{M}(n))(U) .
$$

This implies that we have a surjective morphism

$$
\boldsymbol{k}_{X} \otimes_{\boldsymbol{k}(0)_{X}} \lim _{-\infty \leftarrow n} \mathcal{M}(0) / \mathcal{M}(n) \longrightarrow \lim _{-\infty \leftarrow n} \mathcal{M} / \mathcal{M}(n) .
$$


But it follows once again from [Kashiwara and Rouquier 2008, Lemma 2.11] that

$$
\mathcal{M} \simeq \boldsymbol{k}_{X} \otimes_{\boldsymbol{k}(0)_{X}} M(0) \simeq \boldsymbol{k}_{X} \otimes_{\boldsymbol{k}(0)_{X}} \lim _{-\infty \leftarrow n} M(0) / M(n) .
$$

Thus $\mathcal{M}$ surjects onto $\lim _{-\infty \leftarrow n} \mathcal{M} / \mathcal{M}(n)$. Part (ii) implies this map is also injective.

2E. G-equivariance. Let $G$ be a complex Lie group acting symplectically on $X$, via $T_{g}: X \stackrel{\sim}{\rightarrow} X$ for all $g \in G$. We assume that this action is Hamiltonian with moment map $\mu_{X}: X \rightarrow \mathfrak{g}^{*}$, where $\mathfrak{g}$ is the Lie algebra of $G$.

Definition 2.3. A $G$-action on the $W$-algebra $\mathscr{W}_{X}$ is a $\boldsymbol{k}_{X}$-algebra isomorphism $\rho_{g}: W_{X} \stackrel{\sim}{\longrightarrow} T_{g}^{-1} \mathcal{W}_{X}$ for every $g \in G$ such that $\rho_{g}(a)$ depends holomorphically on $g \in G$ for each section $a \in \mathcal{W}_{X}$ and $\rho_{g_{1}} \circ \rho_{g_{2}}=\rho_{g_{1} g_{2}}$ for all $g_{1}, g_{2} \in G$.

Definition 2.4. Suppose we have fixed a $G$-action on $\mathcal{W}_{X}$. A quasi- $G$-equivariant $\mathscr{W}_{X}$-module is a left $\mathscr{W}_{X}$-module $\mathcal{M}$, together with a $\boldsymbol{k}_{X}$-module isomorphism

$$
\rho_{g}^{\mu}: \mathcal{M} \stackrel{\sim}{\longrightarrow} T_{g}^{-1} \mathcal{M}
$$

for every $g \in G$ such that $\rho_{g}^{M}(m)$ depends holomorphically on $g \in G$ for each section $m \in \mathcal{M}, \rho_{g}^{M} \circ \rho_{h}^{M}=\rho_{g h}^{\mathcal{M}}$ for all $g, h \in G$ and $\rho_{g}^{\mathcal{M}}(a \cdot m)=\rho_{g}(a) \cdot \rho_{g}^{\mathcal{M}}(m)$ for all $g \in G, a \in \mathscr{W}_{X}$ and $m \in \mathcal{M}$.

The category of quasi- $G$-equivariant $\mathscr{W}_{X}$-modules will be denoted $\operatorname{Mod}_{G}\left(\mathscr{W}_{X}\right)$.

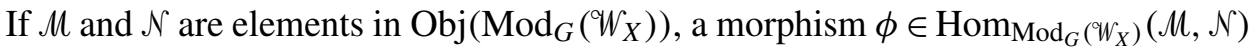
is a collection of morphisms $\phi_{U}: \mathcal{M}(U) \rightarrow \mathcal{N}(U)$ of $\mathscr{W}_{X}(U)$-modules, one for each open set $U \subset X$, that satisfies the usual conditions of being a $\mathscr{W}_{X}$-homomorphism and is such that, for each $g \in G$, the diagram

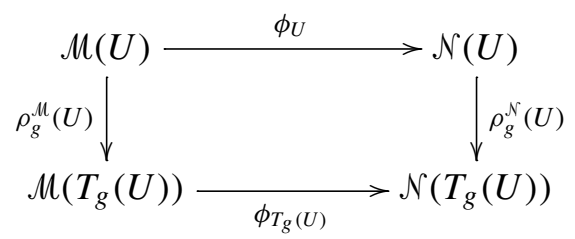

is commutative.

Definition 2.5. Let $G$ act on the algebra $\mathcal{W}_{X}$. A map $\mu_{\mathscr{W}}: \mathfrak{g} \rightarrow \mathcal{W}_{X}(1)$ is said to be a quantized moment map for the $G$-action if $\mu_{W}$ satisfies the following properties:

(i) $\left[\mu_{\mathscr{W}}(A), a\right]=\left.\frac{\mathrm{d}}{\mathrm{d} t} \rho_{\exp (t A)}(a)\right|_{t=0}$,

(ii) $\sigma_{0}(\hbar \mu w(A))=A \circ \mu_{X}$,

(iii) $\mu_{W}(\operatorname{Ad}(g) A)=\rho_{g}\left(\mu_{W}(A)\right)$,

for every $A \in \mathfrak{g}, a \in \mathscr{W}_{X}$ and $g \in G$. 
Let $\mathbb{X}(G):=\operatorname{Hom}_{\mathrm{gp}}\left(G, \mathbb{C}^{*}\right)$ be the lattice of $G$-characters. Note that if $a \in \mathcal{W}_{X}$ is a $\theta$-semiinvariant of $G$ (that is, $\rho_{g}(a)=\theta(g) a$ for all $g \in G$ ), where $\theta \in \mathbb{X}(G)$, then

$$
\left[\mu_{W}(A), a\right]=d \theta(A) a,
$$

where $d: \mathbb{X}(G) \rightarrow\left(\mathfrak{g}^{*}\right)^{G}$ is the differential sending a $G$-character to the corresponding $\mathfrak{g}$-character. From now on we omit the symbol $d$ and think of $\theta \in \mathbb{X}(G)$ as a character for both $G$ and $\mathfrak{g}$. For $\chi \in\left(\mathfrak{g}^{*}\right)^{G}$, we set

$$
\mathscr{L}_{X, \chi}=\mathscr{W}_{X} / \sum_{A \in \mathfrak{g}} \mathscr{W}_{X}\left(\mu_{W}(A)-\chi(A)\right) .
$$

Note that $\mathscr{L}_{X, \chi}$ is a good quasi- $G$-equivariant $\mathscr{W}_{X}$-module, and has lattice

$$
\mathscr{L}_{X, \chi}(0):=\mathscr{W}_{X}(0) / \sum_{A \in \mathfrak{g}} \mathscr{W}_{X}(-1)\left(\mu_{W}(A)-\chi(A)\right) .
$$

We will require the following result, whose proof is based on Holland's result [1999, Proposition 2.4].

Proposition 2.6. Assume that the moment map $\mu_{X}$ is flat. Then, on $X$ we have an isomorphism of graded sheaves

$$
\operatorname{gr}\left(\mathscr{L}_{X, \chi}\right) \simeq \bigoplus_{n \in \mathbb{Z}} \mathcal{O}_{\mu_{X}^{-1}(0)} \hbar^{-n}
$$

Proof. The moment map $\mu_{\mathscr{W}}$ makes $\mathcal{W}_{X}$ into a right $U(\mathfrak{g})$-module. Let $\mathbb{C}_{\chi}$ be the one-dimensional $U(\mathfrak{g})$-module defined by the character $\chi$ so that

$$
\mathscr{L}_{X, \chi}=\mathscr{W}_{X} \otimes_{U(\mathfrak{g})} \mathbb{C}_{\chi} .
$$

As in [Holland 1999, Proposition 2.4], we denote by $B_{\bullet}$ the Chevalley-Eilenberg resolution of $\mathbb{C}_{\chi}$. Thus, $B_{k}=U(\mathfrak{g}) \otimes \wedge^{k} \mathfrak{g}$, and the differential is given by

$$
\begin{aligned}
d_{k}\left(f \otimes x_{1} \wedge \cdots \wedge x_{k}\right)= & \sum_{i=1}^{k}(-1)^{i+1} f\left(x_{i}-\chi\left(x_{i}\right)\right) \otimes x_{1} \wedge \cdots \wedge \hat{x}_{i} \wedge \cdots \wedge x_{k} \\
& +\sum_{1 \leq i<j \leq k}(-1)^{i+j} f \otimes\left[x_{i}, x_{j}\right] \wedge x_{1} \wedge \cdots \wedge \hat{x}_{i} \wedge \cdots \wedge \hat{x}_{j} \wedge \cdots \wedge x_{k} .
\end{aligned}
$$

Then $B_{\bullet}$ is a complex of free $U(\mathfrak{g})$-modules such that $H^{0}\left(B_{\bullet}\right)=\mathbb{C}_{\chi}$ and $H^{k}\left(B_{\bullet}\right)=0$ for $k$ nonzero. Let $C_{\bullet}=\mathscr{W}_{X} \otimes_{U(\mathfrak{g})} B_{\bullet}=W_{X} \otimes \wedge^{\bullet} \mathfrak{g}$. The filtration on $\mathscr{W}_{X}$ induces a

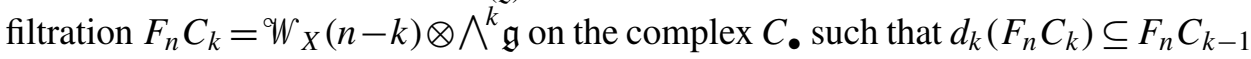
(recall that $\left.\mu \mathscr{w}(\mathfrak{g}) \subset W_{X}(1)\right)$. Note that the filtration is not bounded above or below. However, by Lemma 2.2 the filtration on $C_{\bullet}$ is exhaustive, Hausdorff and complete. We denote by $E_{p, q}^{r}$ the spectral sequence corresponding to the filtration $F_{n}$ on $C_{\bullet}$. Since the filtration is exhaustive, Hausdorff and complete, the proof of [Weibel 
1994, Theorem 5.5.10] shows that the spectral sequence $E$ converges to $H_{\bullet}(C)$ (that the sequence is regular follows from the fact, to be shown below, that it collapses at $\left.E^{1}\right)$. By construction, we have an isomorphism of filtered sheaves $H^{0}(C) \simeq \mathscr{L}_{X, \chi}$ and hence $\operatorname{gr}\left(H_{0}(C)\right) \simeq \operatorname{gr}\left(\mathscr{L}_{X, \chi}\right)$. Denote by $A$ the graded sheaf of algebras $\bigoplus_{n \in \mathbb{Z}} \mathcal{O}_{X} \hbar^{-n}$, where $\mathcal{O}_{X}$ is in degree zero and $\hbar$ has degree -1 . The 0 -th page of the spectral sequence is given by

$$
E_{p, q}^{0}=\mathscr{W}_{X}(p-q) \otimes \bigwedge^{p+q} \mathfrak{g} / W_{X}(p-1-q) \otimes \bigwedge^{p+q} \mathfrak{g} \simeq A_{p-q} \otimes \bigwedge^{p+q} \mathfrak{g} .
$$

Since $\mathbb{C}\left[\mathfrak{g}^{*}\right]$ is a domain and $\mu_{X}$ is assumed to be flat, $\mu_{X}^{*}: \mu_{X}^{-1} \mathfrak{O}_{\mathfrak{g}^{*}} \rightarrow \mathcal{O}_{X}$ is an embedding and we may think of $\mu_{X}^{-1} \mathcal{O}_{\mathfrak{g}^{*}}$ as a subsheaf of $\mathrm{O}_{X}$. Let $x_{1}, \ldots, x_{r}$ be a basis of $\mathfrak{g}$. Then [Bruns and Herzog 1993, Proposition 1.1.2] implies that $\hbar^{-1} x_{1}, \ldots, \hbar^{-1} x_{r}$ form a regular sequence in $A$ at those points where they vanish. By Definition 2.5(ii), the symbol $\sigma_{1}\left(\mu_{W}\left(x_{i}\right)\right)$ equals $\hbar^{-1} x_{i} \in A$. Thus the differential on $E^{0}$ is given by

$$
d_{p+q}\left(f \otimes x_{1} \wedge \cdots \wedge x_{p+q}\right)=\sum_{i=1}^{p+q} f \hbar^{-1} x_{i} \otimes x_{1} \wedge \cdots \wedge \hat{x}_{i} \wedge \cdots \wedge x_{p+q} .
$$

As is explained in [Holland 1999, Proposition 2.4], the only nonzero homology of $E^{0}$ is in the $(p,-p)$ position, where we have

$$
E_{(p,-p)}^{1}=\frac{A_{p}}{A_{p-1} \cdot \hbar \mu_{X}^{*}(\mathfrak{g})} \simeq \mathfrak{O}_{\mu_{X}^{-1}(0)} \hbar^{-p} .
$$

Therefore the sequence collapses at $E^{1}$ and we have

$$
\operatorname{gr}\left(\mathscr{L}_{X, \chi}\right)_{p} \simeq \operatorname{gr}\left(H_{0}(C)\right)_{p} \simeq \mathcal{O}_{\mu_{X}^{-1}(0)} \hbar^{-p},
$$

as required.

2F. $\boldsymbol{F}$-actions. Here we repeat the definition of an $F$-action on $W_{X}$-modules as defined in [Kashiwara and Rouquier 2008]. Let $\mathbb{C}^{\times} \ni t \mapsto T_{t} \in \operatorname{Aut}(X)$ denote an action of the torus $\mathbb{C}^{\times}$on $X$ such that the symplectic 2-form is a semiinvariant of positive weight: $T_{t}^{*} \omega_{X}=t^{m} \omega_{X}$ for some $m>0$.

Definition 2.7. An $F$-action with exponent $m$ on $\mathscr{W}_{X}$ is an action of the group $\mathbb{C}^{\times}$ on $\mathcal{W}_{X}$ as in Definition 2.3 except that $\mathbb{C}^{\times}$also acts on $\hbar$ : if $\mathscr{F}_{t}: \mathscr{W}_{X} \stackrel{\sim}{\rightarrow} T_{t}^{-1} \mathcal{W}_{X}$ denotes the action of $t \in \mathbb{C}^{\times}$then we require that $\mathscr{F}_{t}(\hbar)=t^{m} \hbar$ for all $t \in \mathbb{C}^{\times}$.

It will be convenient to extend the $F$-action of $\mathbb{C}^{\times}$to an action on

$$
\mathscr{W}\left[\hbar^{1 / m}\right]:=\boldsymbol{k}\left(\hbar^{1 / m}\right) \otimes_{\boldsymbol{k}} \mathscr{W}
$$

by setting $\mathscr{F}_{t}\left(\hbar^{1 / m}\right)=t \hbar^{1 / m}$. The category of $F$-equivariant $\mathscr{W}_{X}$-modules will be denoted $\operatorname{Mod}_{F}\left(W_{X}\right)$. As noted in [Kashiwara and Rouquier 2008, §2.3.1], 
$\operatorname{Mod}_{F}\left(\mathcal{W}_{X}\right)$ is an abelian category. Moreover [ibid., §2.3], if there exists a relatively compact open subset $U$ of $X$ such that $\mathbb{C}^{\times} \cdot U=X$ then every good, $F$-equivariant $\mathscr{W}_{X}$-module admits globally a coherent $\mathscr{W}_{X}(0)$-lattice. Such an open set $U$ will exist in the cases we consider. The following lemma will be used later.

Lemma 2.8. Let $\mathcal{M}, \mathcal{N} \in \operatorname{Mod}_{F, G}^{\text {good }}\left(W_{X}\right)$. Assume that $\mathcal{M} \simeq W_{X} / \mathscr{\Phi}$ is a cyclic $\mathscr{W}_{X}$ module, generated by some $G, F$-invariant element, where $\Phi$ is a left ideal generated by finitely many global sections. Then

$$
\operatorname{Hom}_{\operatorname{Mod}_{F, G}^{\mathrm{good}}\left(W_{X}\right)}(M, \mathcal{N})=\operatorname{Hom}_{W_{X}(X)}(M(X), \mathcal{N}(X))^{G, F} .
$$

2G. Example. Let $V$ be an $n$-dimensional vector space. We fix $X=T^{*} V$ with coordinates $x_{1}, \ldots, x_{n}, \xi_{1}, \ldots, \xi_{n}$ and define an action $T_{t}$ of $\mathbb{C}^{\times}$on $X$ such that the corresponding action on coordinate functions is given by $T_{t}\left(x_{i}\right)=t x_{i}$ and $T_{t}\left(\xi_{i}\right)=t \xi_{i}$. Then $T_{t}^{*} \omega_{X}=t^{2} \omega_{X}$. We extend this to an $F$-action on $\mathcal{W}_{T^{*} V}$ by setting $\mathscr{F}_{t}(\hbar)=t^{2} \hbar$. Let $\mathfrak{D}(V)$ denote the ring of algebraic differential operators on $V$.

Lemma 2.9. Taking $F$-invariants in $\mathscr{W}_{T^{*} V}\left(T^{*} V\right)$ gives

$$
\begin{aligned}
\operatorname{End}_{\operatorname{Mod}_{F}\left(W_{T^{*} V}\left[\hbar^{1 / 2}\right]\right)}\left(W_{T^{*} V}\left[\hbar^{1 / 2}\right]\right)^{\text {opp }} & =\mathbb{C}\left[\hbar^{-1 / 2} x_{i}, \hbar^{-1 / 2} \xi_{i}: i \in[1, n]\right] \\
& =\mathbb{C}\left[\hbar^{-1 / 2} x_{i}, \hbar^{1 / 2} \frac{\partial}{\partial x_{i}}: i \in[1, n]\right],
\end{aligned}
$$

where the second equality comes from

$$
\mathfrak{D}(V) \hookrightarrow \mathcal{W}_{T^{*} V}\left(T^{*} V\right), \quad x_{i} \mapsto x_{i} \quad \text { and } \quad \frac{\partial}{\partial x_{i}} \mapsto \hbar^{-1} \xi_{i}
$$

Proof. We can identify $\operatorname{End}_{\operatorname{Mod}_{F}\left(W_{T^{*} V}\left[\hbar^{1 / 2}\right]\right)}\left(W_{T^{*} V}\left[\hbar^{1 / 2}\right]\right)^{\text {opp }}$ with the algebra of $F$-invariant global sections, ${ }^{W_{T} V}\left[\hbar^{1 / 2}\right]\left(T^{*} V\right)^{F}$. Since $T^{*} V$ is connected, taking a power series expansion in a sufficiently small neighborhood of $0 \in T^{*} V$ defines an embedding $\mathrm{O}_{T^{*} V}\left(T^{*} V\right) \hookrightarrow \mathbb{C} \llbracket x_{1}, \ldots, x_{n}, \xi_{1}, \ldots, \xi_{n} \rrbracket$. As $\mathbb{C}^{\times}$-modules, we can identify $\mathscr{W}_{T^{*} V}\left[\hbar^{1 / 2}\right]$ with $\mathbb{O}_{T^{*} V} \widehat{\otimes} \mathbb{C}\left(\left(\hbar^{1 / 2}\right)\right)$ and we get a $\mathbb{C}^{\times}$-equivariant embedding

$$
\mathcal{W}_{T^{*} V}\left[\hbar^{1 / 2}\right]\left(T^{*} V\right) \hookrightarrow \mathbb{C} \llbracket x_{1}, \ldots, x_{n}, \xi_{1}, \ldots, \xi_{n} \rrbracket \widehat{\otimes} \mathbb{C}\left(\left(\hbar^{1 / 2}\right)\right),
$$

where we denote by $\widehat{\otimes}$ the completed tensor product with respect to the linear topology. Taking invariants gives the desired result.

A trivial application of Theorem 3.3 below, with $f=\operatorname{id}_{\mathbb{C}^{n}}$ and $G=\{1\}$, shows

$$
\operatorname{Mod}_{F}\left(\mathcal{W}_{T^{*} V}\left[\hbar^{1 / 2}\right]\right) \simeq \mathbb{C}\left[\hbar^{-1 / 2} x_{i}, \hbar^{1 / 2} \frac{\partial}{\partial x_{i}}: i \in[1, n]\right]-\bmod
$$

\section{3. $W$-affinity}

In this section we give a criterion for the $W$-affinity of a class of $W$-algebras on those symplectic manifolds that are obtained by Hamiltonian reduction. 
3A. The geometric setup. Let $V$ be an $n$-dimensional vector space over $\mathbb{C}$. Its cotangent bundle $T^{*} V$ has the structure of a complex symplectic manifold. Let $G$ be a connected, reductive algebraic group acting algebraically on $V$. This action induces a Hamiltonian action on $T^{*} V$ and we have a moment map

$$
\mu_{T^{*} V}: T^{*} V \longrightarrow \mathfrak{g}^{*}:=(\operatorname{Lie} G)^{*}
$$

such that $\mu_{T^{*} V}(0)=0$. We fix a character $\vartheta \in \mathbb{X}(G)$. Let $\mathfrak{X}$ be the open subset of all $\vartheta$-semistable points in $T^{*} V$ and denote the restriction of $\mu_{T^{*} V}$ to $\mathfrak{X}$ by $\mu_{\mathfrak{X}}$. We assume that

(i) the set $\mu_{\mathfrak{X}}^{-1}(0)$ is nonempty,

(ii) $G$ acts freely on $\mu_{\mathfrak{X}}^{-1}(0)$,

(iii) the moment map $\mu_{T * V}$ is flat.

Set

$$
Y_{\vartheta}:=\mu_{\mathfrak{X}}^{-1}(0) / / G=\operatorname{Proj} \bigoplus_{n \geq 0} \mathbb{C}\left[\mu_{T^{*} V}^{-1}(0)\right]^{n \vartheta}
$$

and write $f: Y_{\vartheta} \rightarrow \mu_{T^{*} V}^{-1}(0) / / G=: Y_{0}$ for the corresponding projective morphism. Condition (i) implies that the categorical quotient $Y_{\vartheta}$ is nonempty. Condition (ii) implies that the morphism $\mu_{\mathfrak{X}}$ is regular at all points in $\mu_{\mathfrak{X}}^{-1}(0)$ and hence $Y_{\vartheta}$ is a nonsingular symplectic manifold. Condition (iii) will be used in Proposition 3.5. We add to our previous assumptions:

(iv) The morphism $f$ is birational and $Y_{0}$ is a normal variety.

In the case of hypertoric varieties, it is shown in Section 4 that assumptions (i)-(iv) hold when the matrix $A$ is unimodular.

Lemma 3.1. Let $\mathcal{O}_{Y_{\vartheta}}^{\mathrm{alg}}$ and $\mathrm{O}_{Y_{0}}^{\mathrm{alg}}$ denote the sheaves of regular functions on $Y_{\vartheta}$ and $Y_{0}$, respectively. If $Y_{\vartheta}, Y_{0}, f$ satisfy assumption (iv) then $\Gamma\left(Y_{\vartheta}, O_{Y_{\vartheta}}^{\mathrm{alg}}\right)=\Gamma\left(Y_{0}, \mathcal{O}_{Y_{0}}^{\mathrm{alg}}\right)$. Proof. It is well-known that the condition implies the statement of the lemma, but we were unable to find any suitable reference, therefore we include a proof for the reader's convenience. For $s \geq 0$, fix $R_{s}=\mathbb{C}\left[\mu_{T^{*} V}^{-1}(0)\right]^{s \vartheta}$ and $R=\bigoplus_{s \geq 0} R_{s}$ so that $Y_{\vartheta}=\operatorname{Proj} R$ and recall that $f$ is the canonical projective morphism from $Y_{\vartheta}$ to $Y_{0}$. By Hilbert's Theorem (see [Kraft 1984, Zusatz 3.2]), $R$ is finitely generated as an $R_{0}$-algebra. Let $x_{1}, \ldots, x_{n} \in R$ be homogeneous generators (of degree at least one) of $R$ as an $R_{0}$-algebra. Then the affine open sets $D_{+}\left(x_{i}\right)=\operatorname{Spec} R_{\left(x_{i}\right)}$ form an open cover of $Y_{\vartheta}$ and

$$
\Gamma\left(Y_{\vartheta}, O_{Y_{\vartheta}}^{\mathrm{alg}}\right)=\bigcap_{i=1}^{n} R_{\left(x_{i}\right)} \subseteq \bigcap_{i=1}^{n} R_{x_{i}},
$$

Let $r \in \Gamma\left(Y_{\vartheta}, \mathcal{O}_{Y_{\vartheta}}^{\mathrm{alg}}\right)$. Then, for each $i$, there exists an $m$ such that $x_{i}^{m} \cdot r \in R$. We choose one $m$ sufficiently large so that $x_{i}^{m} \cdot r \in R$ for all $i$. Since the $x_{i}$ generate 
$R$, we actually have $y \cdot r \in R$ for all $y \in R_{s}$ and $s \geq m_{0}:=n m d$, where $d$ is the maximum of the degrees of $x_{1}, \ldots, x_{n}$. Therefore $y \cdot r \in R$ for all $y \in \bigoplus_{s \geq m_{0}} R_{s}$. Since $r$ has degree zero,

$$
y \cdot r \in \bigoplus_{s \geq m_{0}} R_{S} \quad \text { for all } y \in \bigoplus_{s \geq m_{0}} R_{s} .
$$

Inductively, $y \cdot r^{q} \in \bigoplus_{s \geq m_{0}} R_{s}$ for all $q \geq 1$. Take $y=x_{1}^{m_{0}}$, then $r^{q} \in\left(1 / x_{1}^{m_{0}}\right) R$ for all $q \geq 1$ and hence $R[r] \subset\left(1 / x_{1}^{m_{0}}\right) R$. But, by Hilbert's basis theorem, $R$ is Noetherian and the $R$-module $\left(1 / x_{1}^{m_{0}}\right) R$ is finitely generated, hence the algebra $R[r]$ is finite over $R$. This means $r$ satisfies some monic polynomial $u^{t}+r_{1} u^{t-1}+\cdots+r_{t}$ with coefficients in $R$. However $R$ has degree zero so without loss of generality $r_{i} \in R_{0}$. Thus $r$ is in the integral closure of $R_{0}$ in the degree zero part of the field of fractions of $R$. Now [Hartshorne 1977, Theorem 7.17] says that, since the map $f$ is projective and birational, there exists an ideal $I$ in $R_{0}$ such that $R_{k} \simeq I^{k}$ as $R_{0}$-modules and we have an isomorphism of graded rings $R \simeq \bigoplus_{k \geq 0} I^{k}$. That is, $Y_{\vartheta}$ is isomorphic to the blowup of $Y_{0}$ along $V(I)$. Therefore we can identify the degree zero part of the field of fractions of $R$ with the field of fractions of $R_{0}$. Since $R_{0}$ is assumed to be normal, $r \in R_{0}$ as required.

3B. The quotient morphism will be written $p: \mu_{\mathfrak{X}}^{-1}(0) \rightarrow Y_{\vartheta}$. For each character $\theta \in \mathbb{X}(G)$ and vector space $M$ on which $G$ acts, we denote by $M^{\theta}$ the set of elements $m \in M$ such that $g \cdot m=\theta(g) m$ for all $g \in G$. We can define a coherent sheaf $L_{\theta}$ on the quotient $Y_{\vartheta}$ by $L_{\theta}(U):=\left[0_{\mu_{\mathfrak{X}}^{-1}(0)}\left(p^{-1}(U)\right)\right]^{\theta}$. Since $G$ acts freely on $\mu_{\mathfrak{X}}^{-1}(0)$, $L_{\theta}$ is a line bundle on $Y_{\vartheta}$.

3C. Quantum Hamiltonian reduction. Differentiating the action of $G$ on $V$ produces a morphism of Lie algebras $\mu_{D}: \mathfrak{g} \rightarrow \operatorname{Vect}(V)$, from $\mathfrak{g}$ into the Lie algebra of algebraic vector fields on $V$ :

$$
\mu_{D}(A)(r):=\left.\frac{\mathrm{d}}{\mathrm{d} t} a_{\exp (t A)}^{*}(r)\right|_{t=0},
$$

where $a: G \times V \rightarrow V$ is the action map and $a^{*}: G \times \mathcal{O}(V) \rightarrow \mathcal{O}(V)$ the induced action on functions. We write $\mathfrak{D}(V)$ for the ring of algebraic differential operators on $V$. Since $\operatorname{Vect}(V) \subset \mathfrak{D}(V)$ we get a map $\mu_{D}: \mathfrak{g} \rightarrow \mathfrak{D}(V)$ which extends to an algebra morphism $U(\mathfrak{g}) \rightarrow \mathfrak{D}(V)$. For $\chi \in\left(\mathfrak{g}^{*}\right)^{G}, \theta \in \mathbb{X}$, we define the left $\mathfrak{D}(V)$-module

$$
\mathscr{L}_{D, \chi}:=\mathfrak{D}(V) / \sum_{A \in \mathfrak{g}} \mathfrak{D}(V)\left(\mu_{D}(A)-\chi(A)\right),
$$

and the algebra and $\left(\mathrm{U}_{\chi}, \mathrm{U}_{\chi+\theta}\right)$-bimodule, respectively:

$$
\mathrm{U}_{\chi}=\left(\operatorname{End}_{\mathfrak{D}(V)}\left(\mathscr{L}_{D, \chi}\right)^{G}\right)^{\mathrm{opp}}, \quad \mathrm{U}_{\chi}^{\theta}=\operatorname{Hom}_{\mathfrak{D}(V)}\left(\mathscr{L}_{D, \chi}, \mathscr{L}_{D, \chi+\theta} \otimes \mathbb{C}_{\theta}\right)^{G} .
$$


Fix $\chi \in\left(\mathfrak{g}^{*}\right)^{G}$ and $\theta \in \mathbb{X}$. We consider the following natural homomorphisms:

$$
\begin{array}{ll}
\mathrm{U}_{\chi+\theta}^{-\theta} \otimes_{\mathbb{C}} \mathrm{U}_{\chi}^{\theta} \longrightarrow \mathrm{U}_{\chi+\theta}, & \phi \otimes \psi \mapsto\left(\mathrm{id}_{\chi+\theta} \otimes \mathrm{ev}\right) \circ\left(\psi \otimes \mathrm{id}_{-\theta}\right) \circ \phi, \\
\mathrm{U}_{\chi}^{\theta} \otimes_{\mathbb{C}} \mathrm{U}_{\chi+\theta}^{-\theta} \longrightarrow \mathrm{U}_{\chi}, & \phi \otimes \psi \mapsto\left(\mathrm{id}_{\chi} \otimes \mathrm{ev}\right) \circ\left(\psi \otimes \mathrm{id}_{\theta}\right) \circ \phi,
\end{array}
$$

where $\circ$ is composition of morphisms and ev : $\mathbb{C}_{-\theta} \otimes \mathbb{C}_{\theta} \rightarrow \mathbb{C}$ is the natural map. We write $\chi \rightarrow \chi+\theta$ if the map (4) is surjective and similarly $\chi+\theta \rightarrow \chi$ if the map (5) is surjective. Note that if $\chi+\theta \leftrightarrows \chi$ then, as shown in [McConnell and Robson 2001, Corollary 3.5.4], the algebras $U_{\chi}$ and $U_{\chi+\theta}$ are Morita equivalent.

3D. The sheaf of $W$-algebras. Denote by $\mathscr{W}_{\mathfrak{X}}$ the restriction of the canonical $W$ algebra $\mathscr{W}_{T^{*} V}$ to $\mathfrak{X}$. We define an action of the torus $\mathbb{C}^{\times}$on $T^{*} V$ by $T_{t}(v)=t^{-1} v$ for all $v \in T^{*} V ; \mathfrak{X}$ is a $\mathbb{C}^{\times}$-stable open set. The algebra $\mathscr{W}_{\mathfrak{X}}$ is then equipped with an $F$-action of weight 2 as defined in the setup of Lemma 2.9. Define $\widetilde{\mathcal{W}}_{T^{*} V}:=\mathscr{W}_{T^{*} V}\left[\hbar^{1 / 2}\right]$ and write $\widetilde{\mathcal{W}}_{\mathfrak{X}}$ for its restriction to $\mathfrak{X}$. As noted in Section 2A, we have an embedding $j: \mathfrak{D}(V) \hookrightarrow \mathcal{W}_{T^{*} V}, x_{i} \mapsto x_{i}$ and $\partial / \partial x_{i} \mapsto \hbar^{-1} \xi_{i}$. Composing this morphism with the map $\mu_{D}: \mathfrak{g} \rightarrow \mathfrak{D}(V)$ gives us a map $\mu_{\mathfrak{W}}=j \circ \mu_{D}: \mathfrak{g} \rightarrow \mathscr{W}_{T^{*} V}$. It is a quantized moment map in the sense of Definition 2.5. Then, as in (3), for each $\chi \in\left(\mathfrak{g}^{*}\right)^{G}$, we have defined the $\widetilde{\mathscr{W}}_{T^{*} V}$-module $\mathscr{L}_{T^{*} V, \chi}$. Its restriction to $\mathfrak{X}$ is denoted $\mathscr{L}_{\chi}$. Recall that $\mathscr{L}_{\chi}$ is a good quasi-G-equivariant $\widetilde{W}_{\mathfrak{X}}$-module. If we let $\mathbb{C}^{\times}$act trivially on $\mathfrak{g}$ then the morphism $\mu_{W}$ is $F$-equivariant and hence $\mathscr{L}_{\chi}$ is equipped with an $F$-action. The image of 1 in $\mathscr{L}_{\chi}$ will be denoted by $u_{\chi}$.

3E. Kashiwara and Rouquier [2008] show that one can quantize the process of Hamiltonian reduction to get a family of sheaves of $W$-algebras on $Y_{\vartheta}$ beginning from a $W$-algebra on $T^{*} V$. Set

$$
\mathscr{A}_{\chi}=\left(\left(p_{*} \mathscr{E} n d_{\widetilde{W}_{\mathfrak{X}}}\left(\mathscr{L}_{\chi}\right)\right)^{G}\right)^{\mathrm{opp}} \quad \text { and } \quad \mathscr{A}_{\chi, \theta}=\left(p_{*} \mathcal{H}_{\text {orm }} \widetilde{\mathscr{W}}_{\mathfrak{X}}\left(\mathscr{L}_{\chi}, \mathscr{L}_{\chi+\theta} \otimes \mathbb{C}_{\theta}\right)\right)^{G} \text {, }
$$

where $\theta \in \mathbb{X}(G)$ and $\mathbb{C}_{\theta}$ denotes the corresponding one dimensional $G$-module. By [Kashiwara and Rouquier 2008, Proposition 2.8], $\mathscr{A}_{\chi}$ is a $W$-algebra on $Y_{\vartheta}$ and $\mathscr{A}_{\chi, \theta}$ is a $\left(\mathscr{A}_{\chi}, \mathscr{A}_{\chi+\theta}\right)$-bimodule. Let

$$
\begin{aligned}
\mathscr{A}_{\chi}(0) & =\left(\left(p_{*} \mathscr{E} n d_{\widetilde{\mathbb{W}}_{\mathfrak{X}}(0)}\left(\mathscr{L}_{\chi}(0)\right)\right)^{G}\right)^{\mathrm{opp}}, \\
\mathscr{A}_{\chi, \theta}(0) & =\left(p_{*} \mathscr{H}_{o m_{\widetilde{W}_{X}(0)}}\left(\mathscr{L}_{\chi}(0), \mathscr{L}_{\chi+\theta}(0) \otimes \mathbb{C}_{\theta}\right)\right)^{G},
\end{aligned}
$$

so that $\mathscr{A}_{\chi, \theta}(0)$ is a $\mathscr{A}_{\chi}(0)$-lattice of $\mathscr{A}_{\chi, \theta}$. We have $\mathscr{A}_{\chi}(0) / \mathscr{A}_{\chi}(-1 / 2) \simeq \mathcal{O}_{Y_{\vartheta}}$ and, as noted in [ibid., Proposition 2.8(iii)], $\mathscr{A}_{\chi, \theta}(0) / \mathscr{A}_{\chi, \theta}(-1 / 2) \simeq L_{-\theta}$, where $L_{\theta}$ is the line bundle as defined above. We say that a good $\mathscr{A}_{\chi}$-module $M$ is generated, locally on $Y_{0}$, by its global sections if for each $y \in Y_{0}$ there exists some open neighborhood (in the complex analytic topology) $U \subset Y_{0}$ of $y$ such that the natural map of left $\left(\mathscr{A}_{\chi}\right)_{\mid f^{-1}(U)}$-modules $\left(\mathscr{A}_{\chi}\right)_{\mid f^{-1}(U)} \otimes \mathcal{M}\left(f^{-1}(U)\right) \rightarrow M_{\mid f^{-1}(U)}$ is surjective. 
Definition 3.2. We denote by $\underline{\operatorname{Mod}}_{F}^{\text {good }}\left(\mathscr{A}_{\chi}\right)$ the full subcategory of $\operatorname{Mod}_{F}^{\text {good }}\left(\mathscr{A}_{\chi}\right)$ consisting of all good, $F$-equivariant $\mathscr{A}_{\chi}$-modules $M$ such that:

(i) $\mathcal{M}$ is generated, locally on $Y_{0}$, by its global sections.

(ii) For any nonzero submodule $\mathcal{N}$ of $\mathcal{M}$ in $\operatorname{Mod}_{F}^{\text {good }}\left(\mathscr{A}_{\chi}\right)$ we have

$$
\operatorname{Hom}_{\operatorname{Mod}_{F}^{\text {good }}\left(\mathscr{A}_{\chi}\right)}\left(\mathscr{A}_{\chi}, \mathcal{N}\right) \neq 0 \text {. }
$$

3F. W-affinity. We can now state the main result relating the sheaf of $W$-algebras $A_{\chi}$ on $Y_{\vartheta}$ and the algebra of quantum Hamiltonian reduction $\mathrm{U}_{\chi}$.

Theorem 3.3. Let $\mathscr{A}_{\chi}$ and $\mathrm{U}_{\chi}$ be as above and choose some $\theta \in \mathbb{X}(G)$ such that $L_{\theta}$ is ample.

(i) There is an isomorphism of algebras $\Gamma\left(Y_{\vartheta}, \mathscr{A}_{\chi}\right)^{F} \simeq \mathrm{U}_{\chi}$.

(ii) Assume that we have $\chi \leftarrow \chi+n \theta$ for all $n \in \mathbb{Z}_{\geq 0}$. Then the functor

$$
\mathcal{M} \operatorname{Hom}_{\operatorname{Mod}_{F}^{\operatorname{good}}\left(\mathscr{A}_{\chi}\right)}\left(\mathscr{A}_{\chi}, \mathcal{M}\right)
$$

defines an equivalence of categories $\underline{\operatorname{Mod}}_{F}^{\text {good }}\left(\mathscr{A}_{\chi}\right) \stackrel{\sim}{\longrightarrow} \mathrm{U}_{\chi}$-mod with quasiinverse $M \mapsto \mathscr{A}_{\chi} \otimes \mathrm{u}_{\chi} M$.

(iii) Assume that we have $\chi \leftrightarrows \chi+n \theta$ for all $n \in \mathbb{Z}_{\geq 0}$. Then the functor

$$
\mathcal{M} \mapsto \operatorname{Hom}_{\operatorname{Mod}_{F}^{\text {good }}\left(\mathscr{A}_{\chi}\right)}\left(\mathscr{A}_{\chi}, \mathcal{M}\right)
$$

defines an equivalence of categories $\operatorname{Mod}_{F}^{\text {good }}\left(\mathscr{A}_{\chi}\right) \stackrel{\sim}{\longrightarrow} \mathrm{U}_{\chi}$-mod with quasiinverse $M \mapsto \mathscr{A}_{\chi} \otimes \mathrm{u}_{\chi} M$.

The proof of Theorem 3.3 will occupy the remainder of Section 3.

3G. Proof of the theorem. We fix $\mathscr{A}_{\chi}, \cup_{\chi}$ and $L_{\theta}$ as in Theorem 3.3. First we require some preparatory lemmata. Denote by $\iota$ the embedding $\mathfrak{D}(V) \hookrightarrow \widetilde{\mathcal{W}}_{T^{*} V}\left(T^{*} V\right)$ given by $x_{i} \mapsto \hbar^{-1 / 2} x_{i}$ and $\partial_{i} \mapsto \hbar^{-1 / 2} \xi_{i}$. Equip $\mathfrak{D}(V)$ with a $\frac{1}{2} \mathbb{Z}$-filtration $F_{\bullet} \mathfrak{D}(V)$ by placing $x_{i}$ and $\partial_{i}$ in degree $\frac{1}{2}$ (this is the Bernstein filtration). Then $\iota$ is a strictly filtered embedding in the sense that

$$
\iota\left(F_{k} \mathfrak{D}(V)\right)=\iota(\mathfrak{D}(V)) \cap \widetilde{\mathscr{W}}_{T^{*} V}\left(T^{*} V\right)(k), \quad \text { for all } k \in \frac{1}{2} \mathbb{Z} .
$$

By Lemma 2.9, the image of $\mathfrak{D}(V)$ in $\widetilde{\mathscr{W}}_{T^{*} V}\left(T^{*} V\right)$ is $\widetilde{\mathscr{W}}_{T^{*} V}\left(T^{*} V\right)^{F}$. This implies, since $\mathbb{C}^{\times}$is reductive and $\mu$ w is equivariant, that

$$
\begin{aligned}
\sum_{A \in \mathfrak{g}} \widetilde{W}_{T^{*} V}\left(T^{*} V\right)(\mu \mathscr{W}(A)-\chi(A)) \cap \iota & (\mathfrak{D}(V)) \\
& =\sum_{A \in \mathfrak{g}} \widetilde{W}_{T^{*} V}\left(T^{*} V\right)^{F}\left(\mu_{\mathscr{W}}(A)-\chi(A)\right),
\end{aligned}
$$


which in turn equals

$$
\sum_{A \in \mathfrak{g}} \mathfrak{D}(V)\left(\mu_{D}(A)-\chi(A)\right)
$$

Lemma 3.4 [Ginzburg et al. 2009, Lemma 2.2].

(i) Multiplication in $\mathfrak{D}(V)$ defines an algebra structure on $\left(\mathscr{L}_{D, \chi}\right)^{G}$ such that there is isomorphism of algebras $\mathrm{U}_{\chi} \stackrel{\sim}{\rightarrow}\left(\mathscr{L}_{D, \chi}\right)^{G}$ given by $\phi \mapsto \phi\left(u_{\chi}\right)$ with inverse $f \mapsto r_{f}$, where $r_{f}=\cdot f$ is right multiplication by $f$.

(ii) We have an isomorphism of $\left(\mathrm{U}_{\chi}, \mathrm{U}_{\chi+\theta}\right)$-bimodules $\mathrm{U}_{\chi}^{\theta} \stackrel{\sim}{\longrightarrow}\left(\mathscr{L}_{D, \chi+\theta}\right)^{-\theta}$ given by $\phi \mapsto f$, where $\phi\left(u_{\chi}\right)=f u_{\chi+\theta} \otimes \theta$, with inverse $f u_{\chi+\theta} \mapsto r_{f} \otimes \theta$.

Let us introduce

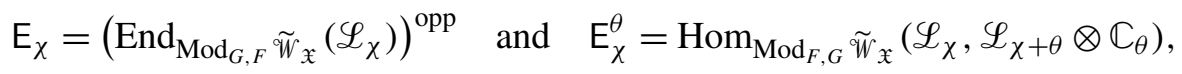

so that $\mathrm{E}_{\chi}^{\theta}$ is a $\left(\mathrm{E}_{\chi}, \mathrm{E}_{\chi+\theta}\right)$-bimodule and $\mathscr{L}_{\chi}$ is a $\left(\widetilde{\mathscr{W}}_{\mathfrak{X}}, \mathrm{E}_{\chi}\right)$-bimodule. By Lemma 2.8, we can identify

$$
\mathrm{E}_{\chi}=\left(\operatorname{End}_{\widetilde{W}_{\mathfrak{X}}(\mathfrak{X})}\left(\mathscr{L}_{\chi}\right)^{G, F}\right)^{\mathrm{opp}} \quad \text { and } \quad \mathrm{E}_{\chi}^{\theta}=\operatorname{Hom}_{\widetilde{W}_{\mathfrak{X}}(\mathfrak{X})}\left(\mathscr{L}_{\chi}, \mathscr{L}_{\chi+\theta} \otimes \mathbb{C}_{\theta}\right)^{G, F} .
$$

Note that (6) implies that the map $\iota$ induces an embedding $\iota: \mathscr{L}_{D, \chi} \hookrightarrow \mathscr{L}_{T^{*} V, \chi}\left(T^{*} V\right)$, and after taking $G, F$-invariants,

$$
\iota: \mathrm{U}_{\chi} \stackrel{\sim}{\longrightarrow}\left(\operatorname{End}_{\widetilde{W}_{T^{*} V}\left(T^{*} V\right)}\left(\mathscr{L}_{T^{*} V, \chi}\right)^{G, F}\right)^{\mathrm{opp}},
$$

and $\mathrm{U}_{\chi}^{\theta} \simeq \operatorname{Hom}_{\widetilde{W}_{T^{*} V}\left(T^{*} V\right)}\left(\mathscr{L}_{T^{*} V, \chi}, \mathscr{L}_{T^{*} V, \chi+\theta} \otimes \mathbb{C}_{\theta}\right)^{G, F}$.

Proposition 3.5. We have a filtered isomorphism $\Psi_{\chi}: U_{\chi} \stackrel{\sim}{\longrightarrow} \mathrm{E}_{\chi}$ in the sense that $\Psi_{\chi}\left(F_{k} \mathrm{U}_{\chi}\right)=F_{k} \mathrm{E}_{\chi}$ for all $k \in \frac{1}{2} \mathbb{Z}$.

Proof. The isomorphism (8) induced by the embedding $\iota$ is filtered in the same sense as $\Psi_{\chi}$ above. Therefore it suffices to show that the natural map

$$
\begin{aligned}
\left(\mathscr{L}_{T^{*} V, \chi}\left(T^{*} V\right)\right)^{G, F}=\left(\operatorname{End}_{\widetilde{W}_{T^{*} V}\left(T^{*} V\right)}\left(\mathscr{L}_{T^{*} V, \chi}\right)^{G, F}\right)^{\text {opp }} & \\
& \longrightarrow\left(\operatorname{End}_{\widetilde{W}_{\mathfrak{X}}(\mathfrak{X})}\left(\mathscr{L}_{\mathfrak{X}, \chi}\right)^{G, F}\right)^{\text {opp }}=\left(\mathscr{L}_{\mathfrak{X}, \chi}(\mathfrak{X})\right)^{G, F}
\end{aligned}
$$

is a filtered isomorphism. The localization morphism $\mathscr{L}_{T^{*} V, \chi}\left(T^{*} V\right) \rightarrow \mathscr{L}_{T^{*} V, \chi}(\mathfrak{X})$ is clearly filtered in the weaker sense that it restricts to a map

$$
\mathscr{L}_{T^{*} V, \chi}\left(T^{*} V\right)(k) \rightarrow \mathscr{L}_{T^{*} V, \chi}(\mathfrak{X})(k)
$$

for each $k \in \frac{1}{2} \mathbb{Z}$. Since the moment map $\mu_{T^{*} V}$ is assumed to be flat, Proposition 2.6 says that the morphism of associated graded spaces is the natural localization map

$$
\bigoplus_{k \in \frac{1}{2} \mathbb{Z}} \mathcal{O}_{\mu_{T^{*} V}^{-1}(0)}\left(T^{*} V\right) \hbar^{-k} \longrightarrow \bigoplus_{k \in \frac{1}{2} \mathbb{Z}} \mathcal{O}_{\mu_{T^{*} V}^{-1}(0)}(\mathfrak{X}) \hbar^{-k}
$$


Note that the filtration on $\mathscr{L}_{T^{*} V, \chi}$ is stable with respect to both $G$ and $F$. Lemma 2.2 says that the globally defined good filtration on $\mathscr{L}_{T^{*} V, \chi}$ is exhaustive and Hausdorff. Therefore, taking invariants with respect to $G$ and $F$, it suffices to show that

$$
\bigoplus_{k \in \frac{1}{2} \mathbb{Z}}\left(\mathcal{O}_{\mu_{T^{*} V}^{-1}(0)}\left(T^{*} V\right) \hbar^{-k}\right)^{G, F} \longrightarrow \bigoplus_{k \in \frac{1}{2} \mathbb{Z}}\left(\mathcal{O}_{\mu_{T^{*} V}^{-1}(0)}(\mathfrak{X}) \hbar^{-k}\right)^{G, F}
$$

is an isomorphism. But, since the $F$-action is contracting,

$$
\left(\mathcal{O}_{\mu_{T^{*} V}^{-1}(0)}\left(T^{*} V\right) \hbar^{-k}\right)^{G, F}=\mathbb{C}\left[\mu_{T^{*} V}^{-1}(0)\right]_{-2 k}^{G},
$$

which is the space of $G$-invariant homogeneous polynomials on $\mu_{T^{*} V}^{-1}(0)$ of degree $-2 k$. Similarly,

$$
\left(\mathbb{O}_{\mu_{T^{*} V}^{-1}(0)}(\mathfrak{X}) \hbar^{-k}\right)^{G, F}=\mathbb{C}\left[\mu_{\mathfrak{X}}^{-1}(0)\right]_{-2 k}^{G} .
$$

Therefore the result follows from Lemma 3.1, which says that

$$
\mathbb{C}\left[\mu_{\mathfrak{X}}^{-1}(0)\right]^{G}=\Gamma\left(Y_{\vartheta}, O_{Y_{\vartheta}}^{\mathrm{alg}}\right)=\Gamma\left(Y_{0}, O_{Y_{0}}^{\mathrm{alg}}\right)=\mathbb{C}\left[\mu_{T^{*} V}^{-1}(0)\right]^{G}
$$

Remark 3.6. In general, it is not true that $\mathrm{U}_{\chi}^{\theta} \simeq \mathrm{E}_{\chi}^{\theta}$ when $\theta \neq 0$.

3H. Shifting. The localization theorem relies on the following result by Kashiwara and Rouquier:

Theorem 3.7 [Kashiwara and Rouquier 2008, Theorem 2.9]. Let $\mathscr{A}_{\chi, \theta}$ and $L_{\theta}$ be as above such that $L_{\theta}$ is ample.

(i) Assume that for all $n \gg 0$, there exists a finite dimensional vector space $W_{n}$ and a split epimorphism of left $\mathscr{A}_{\chi}$-modules $\mathscr{A}_{\chi, n \theta} \otimes W_{n} \rightarrow \mathscr{A}_{\chi}$. Then, for every good $\mathscr{A}_{\chi}$-module $M$, we have $\mathbb{R}^{i} f_{*}(\mathcal{M})=0$ for $i \neq 0$.

(ii) Assume that for all $n \gg 0$ there exists a finite dimensional vector space $U_{n}$ and a split epimorphism of left $\mathscr{A}_{\chi}$-modules $\mathscr{A}_{\chi} \otimes U_{n} \rightarrow \mathscr{A}_{\chi, n \theta}$. Then every good $A_{\chi}$-module is generated, locally on $Y_{0}$, by its global sections.

Lemma 3.8. Let $\mathscr{A}_{\chi}$ and $\mathrm{U}_{\chi}$ be as above and choose $\theta \in \mathbb{X}(G)$.

(i) If $\chi \leftarrow \chi+\theta$ then there exists a finite dimensional vector space $W$ and a split epimorphism $\mathscr{A}_{\chi, \theta} \otimes W \rightarrow \mathscr{A}_{\chi}$.

(ii) If $\chi \rightarrow \chi+\theta$ then there exists a finite dimensional vector space $U$ and a split epimorphism $\mathscr{A}_{\chi} \otimes U \rightarrow \mathscr{A}_{\chi}, \theta$. 
Proof. We begin with (i). Equation (8) implies that we have a morphism $\mathrm{U}_{\chi}^{\theta} \rightarrow \mathrm{E}_{\chi}^{\theta}$, which a direct calculation shows is a morphism of $\left(U_{\chi}, U_{\chi+\theta}\right)=\left(E_{\chi}, E_{\chi+\theta}\right)$ bimodules (here we identify $\mathrm{U}_{\chi}$ with $\mathrm{E}_{\chi}$ via the isomorphism of Proposition 3.5). Thus $\chi \leftarrow \chi+\theta$ implies that $\mathrm{E}_{\chi}^{\theta} \otimes \mathrm{E}_{\chi+\theta}^{-\theta} \rightarrow \mathrm{E}_{\chi}$. Therefore there exists some $k$ and $\phi_{i} \in \mathrm{E}_{\chi}^{\theta}, \psi_{i} \in \mathrm{E}_{\chi+\theta}^{-\theta}$ for $i \in[1, k]$ such that

$$
\left(\mathrm{id}_{\mathscr{L}_{\chi}} \otimes \mathrm{ev}\right) \circ\left(\sum_{i=1}^{k}\left(\psi_{i} \otimes \mathrm{id}_{\mathbb{C}_{\theta}}\right) \circ \phi_{i}\right)=\mathrm{id}_{\mathscr{L}_{\chi}} .
$$

Let $W=\operatorname{Span}_{\mathbb{C}}\left\{\psi_{i}: i \in[1, k]\right\}$ and define $\Psi: \mathscr{L}_{\chi+\theta} \otimes \mathbb{C}_{\theta} \otimes W \rightarrow \mathscr{L}_{\chi}$ by

$$
\Psi(u \otimes \theta \otimes \psi)=\left(\mathrm{id}_{L_{\chi}} \otimes \mathrm{ev}\right)(\psi(u) \otimes \theta) .
$$

The map $\tilde{\Psi}: \mathscr{L}_{\chi} \rightarrow \mathscr{L}_{\chi+\theta} \otimes \mathbb{C}_{\theta} \otimes W$ defined by $v \mapsto \sum_{i=1}^{k} \phi_{i}(v) \otimes \psi_{i}$ is a right inverse to $\Psi$. Hence $\Psi$ is a split epimorphism. Since $\Psi$ and $\tilde{\Psi}$ are $\left(G, \mathbb{C}^{\times}\right)$-equivariant we can apply the functor $p_{*} \mathscr{H}_{\text {om }} \widetilde{\mathscr{W}}_{x}\left(\mathscr{L}_{\chi},-\right)^{G}$, which by [Kashiwara and Rouquier 2008, Proposition 2.8(ii)] is an equivalence, to the morphism $\mathscr{L}_{\chi+\theta} \otimes \mathbb{C}_{\theta} \otimes W \rightarrow \mathscr{L}_{\chi}$ to get the required (necessarily split, epic) morphism.

Part (ii) is similar. Again using Proposition 3.5, $\chi \rightarrow \chi+\theta$ implies that

$$
\mathrm{E}_{\chi+\theta}^{-\theta} \otimes \mathrm{E}_{\chi}^{\theta} \rightarrow \mathrm{E}_{\chi+\theta} .
$$

Therefore there exists some $k$ and $\phi_{i} \in \mathrm{E}_{\chi+\theta}^{-\theta}, \psi_{i} \in \mathrm{E}_{\chi}^{\theta}$ for $i \in[1, k]$ such that

$$
\left(\mathrm{id}_{\mathscr{L}_{\chi+\theta}} \otimes \mathrm{ev}\right) \circ\left(\sum_{i=1}^{k}\left(\psi_{i} \otimes \mathrm{id}_{\mathbb{C}_{-\theta}}\right) \circ \phi_{i}\right)=\operatorname{id}_{\mathscr{L}_{\chi+\theta}} .
$$

Let $U=\operatorname{Span}_{\mathbb{C}}\left\{\psi_{i}: i \in[1, k]\right\}$ and define $\Phi: \mathscr{L}_{\chi} \otimes U \rightarrow \mathscr{L}_{\chi+\theta} \otimes \mathbb{C}_{\theta}$ by $\Phi(u \otimes$ $\psi)=\psi(u)$. The map $\tilde{\Phi}: \mathscr{L}_{\chi+\theta} \otimes \mathbb{C}_{\theta} \rightarrow \mathscr{L}_{\chi} \otimes U$ defined by

$$
v \mapsto\left(\mathrm{id}_{\mathscr{L}_{\chi}} \otimes \mathrm{id}_{U} \otimes \mathrm{ev}\right)\left(\sum_{i=1}^{k} \phi_{i}(v) \otimes \psi_{i}\right)
$$

is a right inverse to $\Phi$. Hence $\Phi$ is a split epimorphism. Since $\Phi$ and $\tilde{\Phi}$ are $\left(G, \mathbb{C}^{\times}\right)$-equivariant we can apply $p_{*} \mathscr{H}_{o m_{\widetilde{W}_{\mathfrak{X}}}}\left(\mathscr{L}_{\chi},-\right)^{G}$ to the morphism

$$
\mathscr{L}_{\chi} \otimes U \rightarrow \mathscr{L}_{\chi+\theta} \otimes \mathbb{C}_{\theta}
$$

to get the required (necessarily split, epic) morphism.

Proof of Theorem 3.3. It follows from the equivalence in [Kashiwara and Rouquier 2008, Proposition 2.8(iv)] that $\Gamma\left(Y_{\vartheta}, \mathscr{A}_{\chi}\right)^{F}=\mathrm{E}_{\chi}$. Therefore part (i) follows from Proposition 3.5. Lemma 3.8 and Theorem 3.7 show that $\chi \leftarrow \chi+n \theta$ for all $n \in \mathbb{Z}_{\geq 0}$ implies that $\mathbb{R}^{i} f_{*}(\mu)=0$ for all $i>0$ and all $M \in \operatorname{Mod}_{F}^{\text {good }}\left(\mathscr{A}_{\chi}\right)$. Similarly, 
$\chi \rightarrow \chi+n \theta$ for all $n \in \mathbb{Z}_{\geq 0}$ implies that every good $\mathscr{A}_{\chi}$-module is generated, locally on $Y_{0}$, by its global sections. Let $o$ denote the image of the origin of $T^{*} V$ in $Y_{0}$. The $\mathbb{C}^{\times}$-action we have defined on $Y_{0}$ (via the $\mathbb{C}^{\times}$-action on $T^{*} V$ ) shrinks every point to $o$, in the sense that $\lim _{t \rightarrow \infty} T_{t}(y)=o$ for all $y \in Y_{0}$. In such a situation, [ibid., Lemma 2.13] says that $\mathbb{R}^{i} f_{*}(\mathcal{M})=0$ for all $i>0$ and all $M \in \operatorname{Mod}_{F}^{\text {good }}\left(\mathscr{A}_{\chi}\right)$ implies that $\operatorname{Hom}_{\operatorname{Mod}_{F}^{\text {good }}\left(\mathscr{A}_{\chi}\right)}\left(\mathscr{A}_{\chi},-\right)$ is an exact functor. Similarly, [ibid., Lemma 2.14] says that if every good $\mathscr{A}_{\chi}$-module $M$ is generated, locally on $Y_{0}$, by its global sections then every $\mathcal{M}$ is generated by its $F$-invariant global sections. That is,

$$
\mathscr{A}_{\chi} \otimes \mathrm{U}_{\chi} \operatorname{Hom}_{\operatorname{Mod}_{F}^{\text {good }}\left(\mathscr{A}_{\chi}\right)}\left(\mathscr{A}_{\chi}, M\right) \rightarrow M
$$

With these facts, one can follow the proof of [Hotta et al. 2008, Corollary 11.2.6], more or less word for word.

\section{Hypertoric varieties}

4A. As we have seen in the previous section, when one has a reductive group $G$ acting on a vector space $V$, there exists a family of $W$-algebras on the Hamiltonian reduction of the cotangent bundle of $V$. The simplest such situation is where $G=\mathbb{T}$, a $d$-dimensional torus. In this case the corresponding Hamiltonian reduction is called a hypertoric variety. In this section we recall the definition of, and basic facts about, hypertoric varieties. The reader is advised to consult [Proudfoot 2008] for an excellent introduction to hypertoric varieties. Here we will follow the algebraic presentation given in [Hausel and Sturmfels 2002]. Thus, in this section only, spaces will be algebraic varieties over $\mathbb{C}$ in the Zariski topology.

4B. Torus actions. Fix $1 \leq d<n \in \mathbb{N}$ and let $\mathbb{T}:=\left(\mathbb{C}^{\times}\right)^{d}$. We consider $\mathbb{T}$ acting algebraically on the $n$-dimensional vector space $V$. If we fix coordinates on $V$ such that the corresponding coordinate functions $x_{1}, \ldots, x_{n}$ are eigenvectors for $\mathbb{T}$ then the action of $\mathbb{T}$ is encoded by a $d \times n$ integer valued matrix

$$
A=\left[a_{1}, \ldots, a_{n}\right]=\left(a_{i j}\right)_{i \in[1, d], j \in[1, n]},
$$

and is given by $\left(\xi_{1}, \ldots, \xi_{d}\right) \cdot x_{i}=\xi_{1}^{a_{1 i}} \ldots \xi_{d}^{a_{d i}} x_{i}$ for all $\left(\xi_{1}, \ldots, \xi_{d}\right) \in \mathbb{T}$. We fix the coordinate ring of $V$ to be $R:=\mathbb{C}\left[x_{1}, \ldots, x_{n}\right]$. The algebra $R$ is graded by the action of $\mathbb{T}, \operatorname{deg}\left(x_{i}\right)=a_{i}$. We make the assumption that the $d \times d$ minors of $A$ are relatively prime. This ensures that the map $\mathbb{Z}^{n} \stackrel{A}{\longrightarrow} \mathbb{Z}^{d}$ is surjective and hence the stabilizer of a generic point is trivial.

4C. Since $\mathbb{Z}^{d}$ is a free $\mathbb{Z}$-module, the above assumption implies that we can choose an $n \times(n-d)$ integer valued matrix $B=\left[b_{1}, \ldots, b_{n}\right]^{T}$ so that the following sequence is exact:

$$
0 \longrightarrow \mathbb{Z}^{n-d} \stackrel{B}{\longrightarrow} \mathbb{Z}^{n} \stackrel{A}{\longrightarrow} \mathbb{Z}^{d}=\mathbb{X} \longrightarrow 0,
$$


where, as before, $\mathbb{X}:=\operatorname{Hom}_{g p}\left(\mathbb{T}, \mathbb{C}^{\times}\right)$is the character lattice of $\mathbb{\mathbb { V }}$ and $\mathbb{Z}^{n}$ is identified with the character lattice of $\left(\mathbb{C}^{\times}\right)^{n} \subset \mathrm{GL}\left(\mathbb{C}^{n}\right)$. The dual $\operatorname{Hom}_{\mathbb{Z}}(\mathbb{X}, \mathbb{Z})$ of $\mathbb{X}$, which parametrizes one-parameter subgroups of $\mathbb{\mathbb { T }}$, will be denoted $\mathbb{Y}$. Applying the functor $\operatorname{Hom}\left(\cdot, \mathbb{C}^{\times}\right)$to the sequence $(9)$ gives a short exact sequence of abelian groups

$$
1 \longrightarrow \mathbb{T} \stackrel{A^{T}}{\longrightarrow}\left(\mathbb{C}^{\times}\right)^{n} \stackrel{B^{T}}{\longrightarrow}\left(\mathbb{C}^{\times}\right)^{n-d} \longrightarrow 1 .
$$

Let $\mathfrak{t}$ denote the Lie algebra of $\mathbb{T}$ and $\mathfrak{g}$ the Lie algebra of $\left(\mathbb{C}^{\times}\right)^{n}$. Differentiating the sequence (10) produces the short exact sequence

$$
0 \longrightarrow \mathfrak{t} \stackrel{A^{T}}{\longrightarrow} \mathfrak{g} \stackrel{B^{T}}{\longrightarrow} \operatorname{Lie}\left(\mathbb{C}^{\times}\right)^{n-d} \longrightarrow 0
$$

of abelian Lie algebras.

4D. Geometric invariant theory. The standard approach to defining "sensible" algebraic quotients of $V$ by $\mathbb{T}$ is to use geometric invariant theory. We recall here the basic construction that will be used. Let $\mathbb{X}_{\mathbb{Q}}:=\mathbb{X} \otimes_{\mathbb{Z}} \mathbb{Q}$ be the space of fractional characters. We fix a stability parameter $\delta \in \mathbb{X}_{\mathbb{Q}}$. For $\underline{k}=\left(k_{1}, \ldots, k_{n}\right) \in \mathbb{N}^{n}$, the monomial $x_{1}^{k_{1}} \cdots x_{n}^{k_{n}}$ will be written $x^{\underline{k}}$. Then $\lambda \cdot x^{\underline{k}}=\lambda^{A \cdot \underline{k}} x^{\underline{k}}$ and we define

$$
R^{\delta}:=\operatorname{Span}_{\mathbb{C}}\left(x^{\underline{k}} \mid A \cdot \underline{k}=\delta\right)
$$

to be the space of $\mathbb{T}$-semiinvariants of weight $\delta$. Note that $R^{\delta}=0$ if $\delta \notin \mathbb{X}$. A point $p \in V$ is said to be $\delta$-semistable if there exists an $n>0$ such that $n \delta \in \mathbb{X}$ and $f \in R^{n \delta}$ with $f(p) \neq 0$. A point $p$ is called $\delta$-stable if it is $\delta$-semistable and in addition its stabilizer under $\mathbb{T}$ is finite. The set of $\delta$-semistable points in $V$ will be denoted $V_{\delta}^{\text {ss }}$. The parameter $\delta$ is said to be effective if $R^{n \delta} \neq 0$ for some $n>0$ (by the Nullstellensatz this is equivalent to $V_{\delta}^{\text {ss }} \neq \varnothing$ ).

Definition 4.1. Let $\delta \in \mathbb{X}_{\mathbb{Q}}$ be an effective stability condition. The G.I.T quotient of $V$ by $\mathbb{T}$ with respect to $\delta$ is the variety

$$
X(A, \delta):=\operatorname{Proj} \bigoplus_{k \geq 0} R^{k \delta}
$$

it is projective over the affine quotient $X(A, 0):=\operatorname{Spec}\left(R^{\mathbb{T}}\right)$.

If a point $p \in V$ is not $\delta$-semistable it is called $\delta$-unstable. Using the oneparameter subgroups of $\mathbb{T}$ one can describe the set $V_{\delta}^{\text {us }}$ of $\delta$-unstable points. We denote by $\langle\cdot, \cdot\rangle$ the natural pairing between $\mathbb{Y}$ and $\mathbb{X}$ (and by extension between $\mathfrak{t}$ and $\left.\mathfrak{t}^{*}\right)$. Let $V\left(f_{1}, \ldots, f_{k}\right)$ denote the set of common zeros of the polynomials $f_{1}, \ldots, f_{k} \in R$. 
Lemma 4.2. Let $\delta \in \mathbb{X}_{\mathbb{Q}}$ be an effective stability parameter. The $\delta$-unstable locus is

$$
V_{\delta}^{\mathrm{us}}=\bigcup_{\substack{\lambda \in \mathbb{V} \\\langle\lambda, \delta\rangle<0}} V\left(x_{i} \mid\left\langle\lambda, a_{i}\right\rangle<0\right) .
$$

Moreover, there exists a finite set $\mathscr{F}(\delta)=\left\{\lambda_{1}, \ldots, \lambda_{k}\right\} \subset \mathbb{Y},\left\langle\lambda_{i}, \delta\right\rangle<0$ such that

$$
\bigcup_{\substack{\lambda \in \mathbb{Y} \\\langle\lambda, \delta\rangle<0}} V\left(x_{i} \mid\left\langle\lambda, a_{i}\right\rangle<0\right)=\bigcup_{\lambda \in \mathscr{F}(\delta)} V\left(x_{i} \mid\left\langle\lambda, a_{i}\right\rangle<0\right) .
$$

Proof. Let $S:=R[t]$ and extend the action of $\mathbb{\rrbracket}$ from $R$ to $S$ by setting $g \cdot t=\delta(g)^{-1} t$ for all $g \in \mathbb{T}$. Then $(S)^{\mathbb{T}}=\bigoplus_{n \geq 0} R^{n \delta} \cdot t^{n}$. Now

$$
\begin{aligned}
u \in V_{\delta}^{\text {us }} & \Longleftrightarrow f(u)=0 \quad \text { for all } f \in R^{n \delta}, n>0, \\
& \Longleftrightarrow F(u, 1)=0 \quad \text { for all } F \in\left(S^{\mathbb{T}}\right)_{+}=\left(S_{+}\right)^{\mathbb{T}}, \\
& \Longleftrightarrow \overline{\mathbb{T} \cdot(u, 1)} \cap V \times\{0\} \neq \varnothing,
\end{aligned}
$$

where $\left(S^{\mathbb{T}}\right)_{+}=\left(S_{+}\right)^{\mathbb{T}}$ follows from the fact that $\mathbb{T}$ is reductive. Then [Kempf 1978, Theorem 1.4] says that there exists a one-parameter subgroup $\lambda \in \mathbb{Y}$ such that $\lim _{t \rightarrow 0} \lambda(t) \cdot(u, 1) \in V \times\{0\}$. Writing $u=u_{1}+\cdots+u_{n}$ such that $x_{i}(u)=u_{i}$, we have

$$
\lambda(t) \cdot(u, 1)=\left(\sum_{i=1}^{n} t^{-\left\langle\lambda, a_{i}\right\rangle} u_{i}, t^{\langle\lambda, \delta\rangle}\right),
$$

which implies that $u_{i}=0$ for all $i \in[1, n]$ such that $\left\langle\lambda, a_{i}\right\rangle>0$ and $\langle\lambda, \delta\rangle>0$. This shows that the left hand side of (12) is contained in the right hand side. Conversely, if $u$ is $\delta$-semistable then it is also $\phi$-semistable with respect to the action of the one dimensional torus $\lambda: \mathbb{T} \hookrightarrow \mathbb{T}$ on $V$, where $\phi$ is the character of $\mathbb{T}$ defined by $t \mapsto t^{\langle\lambda, \delta\rangle}$.

4E. The variety $X(A, \delta)$ is a toric variety and, as shown in [Hausel and Sturmfels 2002, Corollary 2.7], any semiprojective toric variety equipped with a fixed point is isomorphic to $X(A, \delta)$ for suitable $A$ and $\delta$. Fix $S \subset V$ and let $\delta_{1}, \delta_{2} \in \mathbb{X}_{\mathbb{Q}}$ be two stability parameters such that $S_{\delta_{1}}^{\mathrm{ss}}, S_{\delta_{2}}^{\mathrm{ss}} \neq 0$. Then $\delta_{1}$ and $\delta_{2}$ are said to be equivalent if $S_{\delta_{1}}^{\mathrm{ss}}=S_{\delta_{2}}^{\mathrm{ss}}$. The set of all $\rho$ equivalent to a fixed $\delta$ will be denoted $C(\delta)$. These equivalence classes form the relative interiors of the cones of a rational polyhedral fan $\Delta(\mathbb{T}, S)$, called the G.I.T. fan, in $\mathbb{X}_{\mathbb{Q}}$. The support of $\Delta(\mathbb{T}, S)$ is the set of all effective $\delta \in \mathbb{X}_{\mathbb{Q}}$ such that $S_{\delta}^{\text {ss }} \neq 0$ and is denoted $|\Delta(\mathbb{T}, S)|$. We will mainly be concerned with $S=V$. The cones in $\Delta(\mathbb{T}, V)$ having the property that the stable locus is properly contained in the semistable locus are called the walls of $\Delta(\mathbb{T}, V)$. The G.I.T. fan is quite difficult to describe explicitly; see [Oda and Park 1991]. However one has the following explicit description of the walls of $\Delta(\mathbb{T}, V)$. 
Lemma 4.3. Let $\mathbb{1}$ act on $V$ via $A$ as in Section $4 B$. Then $|\Delta(\mathbb{T}, V)|=\sum_{i=1}^{n} \mathbb{Q}_{\geq 0} \cdot a_{i}$ and the walls of the fan are $\sum_{i \in J} \mathbb{Q}_{\geq 0} \cdot a_{i}$, where $J \subset[1, n]$ is any subset such that $\operatorname{dim}_{\mathbb{Q}}\left(\operatorname{Span}_{\mathbb{Q}}\left(a_{i} \mid i \in J\right)\right)=d-1$.

Proof. Let $0 \neq \delta \in \sum_{i=1}^{n} \mathbb{Z}_{\geq 0} \cdot a_{i}$ and write $\delta=\sum_{i \in I} n_{i} a_{i}$ where $I \subset[1, n]$ and $n_{i}>0$ for all $i \in I$. Then $0 \neq f=\prod_{i \in I} x_{i}^{n_{i}} \in R^{\delta}$ implies that $\delta$ is effective. Now let $\delta \in \mathbb{X}$ be any effective stability parameter and choose $0 \neq p \in V_{\delta}^{\text {ss }}$. Write $p=p_{1}+\cdots+p_{n}$ so that $x_{i}(p)=p_{i}$ and let $I=\left\{i \in[1, n] \mid p_{i} \neq 0\right\}$. Then Lemma 4.2 shows that

$$
\begin{aligned}
p \in V_{\delta}^{\text {ss }} & \Longleftrightarrow\left(\langle\lambda, \delta\rangle<0 \Rightarrow \text { there exists } i \in I \text { such that }\left\langle\lambda, a_{i}\right\rangle<0\right) \\
& \Longleftrightarrow\{\lambda \in \mathbb{Y} \mid\langle\lambda, \delta\rangle<0\} \cap\left(\sum_{i \in I} \mathbb{Z}_{\geq 0} \cdot a_{i}\right)^{\vee}=\varnothing \\
& \Longleftrightarrow\left(\sum_{i \in I} \mathbb{Z}_{\geq 0} \cdot a_{i}\right)^{\vee} \subset\{\lambda \in \mathbb{Y} \mid\langle\lambda, \delta\rangle \geq 0\} \\
& \Longleftrightarrow \delta \in \sum_{i \in I} \mathbb{Z}_{\geq 0} \cdot a_{i} .
\end{aligned}
$$

Now choose $\delta \in \mathbb{X}$ to lie on a wall. By definition, there exists a $\delta$-semistable point $p$ such that $\operatorname{dim} \operatorname{Stab}_{\mathbb{T}}(p) \geq 1$. Let $I$ be as above. Then $\operatorname{dim} \operatorname{Stab}_{\mathbb{T}}(p) \geq 1 \mathrm{implies}$ that the subspace $\sum_{i \in I} \mathbb{Q} \cdot a_{i}$ must be a proper subspace of $\mathbb{X}_{\mathbb{Q}}$. The above reasoning shows that $\delta \in \sum_{i \in I} \mathbb{Z}_{\geq 0} \cdot a_{i}$ as required.

Lemma 4.3 shows that, under our assumption on $A$, the maximal cones of $\Delta(\mathbb{T}, V)$ are all $d$-dimensional. We will refer to these maximal cones as the $d$ cones of $\Delta(\mathbb{T}, V)$. The integer valued matrix $A$ is said to be unimodular ${ }^{1}$ if every $d \times d$ minor of $A$ takes values in $\{-1,0,1\}$. Combining [Hausel and Sturmfels 2002, Corollary 2.7 and Corollary 2.9] gives the following theorem:

Theorem 4.4. The variety $X(A, \delta)$ is an orbifold if and only if $\delta$ belongs to the interior of a $d$-cone of $\Delta(\mathbb{T}, V)$. It is a smooth variety if and only if $\delta$ belongs to the interior of a $d$-cone of $\Delta(\mathbb{T}, V)$ and $A$ is unimodular.

4F. Hypertoric varieties. Define $A^{ \pm}:=[A,-A]$, a $d \times 2 n$ matrix. It defines a grading on the ring $R:=\mathbb{C}\left[T^{*} V\right]=\mathbb{C}\left[x_{1}, \ldots, x_{n}, y_{1}, \ldots, y_{n}\right]$. For $\delta$ in the interior of a $d$-cone of $\Delta\left(\mathbb{T}, T^{*} V\right)$, the corresponding toric variety $X\left(A^{ \pm}, \delta\right)$ is called a Lawrence toric variety associated to $A$. It is a G.I.T. quotient of the symplectic vector space $T^{*} V$ with canonical symplectic form

$$
\omega=d x_{1} \wedge d y_{1}+\cdots+d x_{n} \wedge d y_{n} .
$$

${ }^{1}$ In [Hausel and Sturmfels 2002], the authors define $A$ to be unimodular if every nonzero $d \times d$ minor of $A$ has the same absolute value. However they also, as do we, make the assumption that the $d \times d$ minors of $A$ are relatively prime. Thus, their definition agrees with ours. 
The action of $\mathbb{T}$ is Hamiltonian and the moment map is given by

$$
\mu: T^{*} V \longrightarrow \mathfrak{t}^{*}, \quad \mu(\boldsymbol{x}, \boldsymbol{y})=\left(\sum_{j=1}^{n} a_{i j} x_{j} y_{j}\right)_{i \in[1, d]} .
$$

Consider the ideal

$$
I:=I\left(\mu^{-1}(0)\right)=\left\langle\sum_{j=1}^{n} a_{i j} x_{j} y_{j} \mid i \in[1, d]\right\rangle \subset R ;
$$

it is homogeneous and generated by $\mathbb{T}$-invariant polynomials.

Definition 4.5. The hypertoric variety associated to $A$ and $\delta$ is defined to be

$$
Y(A, \delta):=\mu^{-1}(0) / / \delta \mathbb{T}=\operatorname{Proj} \bigoplus_{k \geq 0}(R / I)^{k \delta} ;
$$

it is projective over the affine quotient $Y(A, 0):=\operatorname{Spec}\left((R / I)^{\mathbb{T}}\right)$.

The basic properties of hypertoric varieties can be summarized as follows:

Proposition 4.6 [Hausel and Sturmfels 2002, Proposition 6.2]. If $\delta$ is in the interior of a $d$-cone of $\Delta\left(\mathbb{T}, \mu^{-1}(0)\right)$ then the hypertoric variety $Y(A, \delta)$ is an orbifold. It is smooth if and only if $\delta$ is in the interior of a $d$-cone of $\Delta\left(\mathbb{T}, \mu^{-1}(0)\right)$ and $A$ is unimodular.

4G. In this subsection we show that the assumptions of Section $3 \mathrm{~A}$ are valid for hypertoric varieties. Let $f: Y(A, \delta) \rightarrow Y(A, 0)$ be the projective morphism from $Y(A, \delta)$ to $Y(A, 0)$. Lemma 4.9 below together with Proposition 4.6 implies that $f$ is birational and hence a resolution of singularities when $Y(A, \delta)$ is smooth. The symplectic form $\omega$ on $T^{*} V$ induces a symplectic 2-form on the smooth locus of $Y(A, \delta)$. In particular, when $Y(A, \delta)$ is smooth it is a symplectic manifold. Proposition 4.11 below shows that $Y(A, 0)$ is a symplectic variety and the resolution $f$ is a symplectic resolution. ${ }^{2}$ This implies that $Y(A, 0)$ is also normal.

Lemma 4.7. The moment map is flat and $\mu^{-1}(0)$ is a reduced complete intersection in $T^{*} V$. If no row of the matrix $B$ is zero then $\mu^{-1}(0)$ is irreducible.

Proof. The graded lexicographic ordering on a monomial $\underline{x}^{\alpha}, \alpha \in \mathbb{Z}^{2 n}$, is defined by saying that $\underline{x}^{\alpha}>\underline{x}^{\beta}$ if and only if $|\alpha|>|\beta|$, or $|\alpha|=|\beta|$ and the leftmost nonzero entry of $\alpha-\beta$ is positive; see [Cox et al. 2007, page 58]. After permuting the variables $x_{1}, \ldots, x_{n}$, we may assume that the first $d$ columns of $A$ are linearly independent. Applying an automorphism of $\mathbb{T}$ and then letting $\mathbb{T}$ act is the same as multiplying $A$ on the right by some unimodular $d \times d$-matrix. Using this fact we

${ }^{2}$ We refer the reader to [Fu 2006] for the definition of symplectic variety and symplectic resolution. 
may assume that the leftmost $d \times d$-block of $A$ is the identity matrix. This allows us to rewrite the generators of $I$ as

$$
\left\{x_{i} y_{i}-\sum_{j=d+1}^{n} c_{i, j} x_{j} y_{j} \mid i=1, \ldots, d\right\}, \quad \text { where } c_{i, j} \in \mathbb{Z} .
$$

By [Cox et al. 2007, Theorem 8, page 461], $\operatorname{dim} \mu^{-1}(0)=\operatorname{dim} V(\operatorname{in}(I))$, where in $(I)$ denotes the initial ideal of $I$ with respect to the ordering $x_{1}>x_{2}>\cdots>y_{1}>y_{2} \cdots$. Now by [Cox et al. 2007, Theorem 3 (Division algorithm), page 64], we have $\operatorname{in}(I)=\left\langle x_{1} y_{1}, \ldots, x_{d} y_{d}\right\rangle$. This is the zero set of a union of $2^{d}$ linear subspaces of $T^{*} V$ of dimension $2 n-d$. Therefore $\operatorname{dim} \mu^{-1}(0)=2 n-d$ and it follows from [Holland 1999, Lemma 2.3] that the moment map is flat. To prove that it is a complete intersection we must show that the generators of $I$ given above form a regular sequence in the polynomial ring $R$. Once again, it suffices to note that $x_{1} y_{1}, \ldots, x_{d} y_{d}$ is a regular sequence. Also, since the ideal in $(I)$ is radical, the ideal $I$ is itself radical.

Now note that, since the sequence (9) is exact, the matrix $B$ contains a row of zeros if and only if there exists an $i \in[1, \ldots, d]$ such that $c_{i, j}=0$ for all $j>d$. So, when $B$ contains no rows equal to zero we can write

$$
y_{i}=x_{i}^{-1} \sum_{j=d+1}^{n} c_{i, j} x_{j} y_{j} \quad \bmod I
$$

on the open set $\mu^{-1}(0) \backslash V\left(x_{1} \cdots x_{d}\right)$. This shows that $\mu^{-1}(0)$ contains an open set isomorphic to $\mathbb{A}^{2 n-d}$. We just need to show that this open set is dense. Since $\mu^{-1}(0)$ is a complete intersection, it is pure dimensional. Therefore it suffices to show that the dimension of $\mu^{-1}(0) \cap V\left(x_{1} \cdots x_{d}\right)$ is at most $2 n-d-1$. Consider $Y=\mu^{-1}(0) \cap V\left(x_{1}\right)$ and let $J=I(Y)$. We may assume without loss of generality that $c_{1, d+1} \neq 0$. Then $J$ is generated by $x_{1}, x_{i} y_{i}-\sum_{j=d+2}^{n} c_{i, j} x_{j} y_{j}$ for $j=2, \ldots, d$ and $x_{d+1} y_{d+1}+\sum_{j=d+2}^{n} c_{1, j} x_{j} y_{j}$. Hence in $(J)=\left\langle x_{1}, x_{2} y_{2}, \ldots, x_{d+1} y_{d+1}\right\rangle$, which defines a variety of dimension $2 n-d-1$ as required.

From now on we assume that no row of the matrix $B$ is zero.

Lemma 4.8. For any $A$, we have $\operatorname{dim} X\left(A^{ \pm}, 0\right)=2 n-d$.

Proof. Let $U=V \backslash V\left(x_{1} \cdots x_{n}\right)$ and let $S_{1}=\mathbb{C}\left[x_{1}^{ \pm 1}, \ldots, x_{n}^{ \pm 1}\right]^{\mathbb{T}}$ denote the coordinate ring of the quotient $U / \mathbb{T}$. Let $F_{1}$ be the field of fractions of $S_{1}$. Let $S_{2}=\mathbb{C}\left[X\left(A^{ \pm}, 0\right)\right]$ and $F_{2}$ its field of fractions. We claim that $F_{1} \subset F_{2}$. An element in $F_{1}$ is a fraction $f\left(x_{1}, \ldots, x_{n}\right) / g\left(x_{1}, \ldots, x_{n}\right)$, where $f$ and $g$ are homogeneous of the same weight with respect to $\mathbb{T}$. Then $f(\boldsymbol{x}) f(\boldsymbol{y}), g(\boldsymbol{x}) f(\boldsymbol{y}) \in S_{2}$ and $f(\boldsymbol{x}) f(\boldsymbol{y}) / g(\boldsymbol{x}) f(\boldsymbol{y})=f(\boldsymbol{x}) / g(\boldsymbol{x})$ as required. Since $\operatorname{dim} \mathbb{T}^{n} / \mathbb{T}=n-d$, to prove the lemma it suffices to show that the field extension $F_{1} \subset F_{2}$ has transcendental 
degree $n$. Consider the field $K=F_{1}\left\langle x_{1} y_{1}, \ldots, x_{n} y_{n}\right\rangle$. Then $F_{1} \subset K \subset F_{2}$ and $K$ is a purely transcendental extension of $F_{1}$ of degree $n$. We claim that $K=F_{2}$. To show this it is sufficient to show that if $f \in S_{2}$ is a polynomial in the $x_{i}$ and $y_{j}$ then $f \in K$. We show more generally that if $f=f_{1} / g$, where $f_{1}, g \in S_{2}$ and $g$ a monomial, then $f \in K$. We prove the claim by induction on the number of terms in $f$ (note that even though there is some choice in the exact form of each of the terms in $f$, the number of terms is unique). Let $u=\alpha x^{i} y^{j}, \boldsymbol{i}, \boldsymbol{j} \in \mathbb{Z}^{n}$, be some nonzero term of $f$. Then $(x y)^{-j} u \in F_{1}$ and $(x y)^{-j} f-(x y)^{-j} u \in K$ by induction. Since $(x y)^{-j} \in K$, this implies that $f \in K$.

Note that, unlike $X\left(A^{ \pm}, 0\right)$, the dimension of $X(A, 0)$ can vary greatly depending on the specific entries of $A$.

Lemma 4.9. For any $A$, we have $\operatorname{dim} Y(A, 0)=2(n-d)$ and $Y(A, 0)$ is CohenMacaulay.

Proof. By Hochster's Theorem [Bruns and Herzog 1993, Theorem 6.4.2], the ring $\mathbb{C}\left[X\left(A^{ \pm}, 0\right)\right]$ is Cohen-Macaulay. As noted in Lemma 4.7, the generators $u_{1}, \ldots, u_{d}$ of $I$ form a regular sequence in $R$. Since $\mathbb{T}$ is reductive,

$$
R=\mathbb{C}\left[X\left(A^{ \pm}, 0\right)\right] \oplus E
$$

as a $\mathbb{C}\left[X\left(A^{ \pm}, 0\right)\right]$-module. Therefore projection from $R$ to $\mathbb{C}\left[X\left(A^{ \pm}, 0\right)\right]$ is a Reynolds operator in the sense of [ibid., page 270]. Since $u_{1}, \ldots, u_{d}$ are $\mathbb{T}$ invariant, [ibid., Proposition 6.4.4] now says that they form a regular sequence in $X\left(A^{ \pm}, 0\right)$. Therefore [ibid., Theorem 2.1.3] says that $Y(A, 0)$ is Cohen-Macaulay with $\operatorname{dim} Y(A, 0)=\operatorname{dim} X\left(A^{ \pm}, 0\right)-d$. The lemma follows from Lemma 4.8.

Lemma 4.10. Let $\delta \in \mathbb{X}_{\mathbb{Q}}$. The graded ring $\bigoplus_{k \geq 0}(R / I)^{k \delta}$ is Cohen-Macaulay, that is, $Y(A, \delta)$ is arithmetically Cohen-Macaulay.

Proof. Consider $S=R[t]$ with $\mathbb{T}$ acting on $t$ via $g \cdot t=\delta(g)^{-1} t$. Replacing $R$ with $S$ in the proof of Lemma 4.9 gives a proof of the statement.

Proposition 4.11. Let $A$ be unimodular and choose $\delta$ in the interior of $a d$ cone of $\Delta\left(\mathbb{T}, \mu^{-1}(0)\right)$. Then $Y(A, 0)$ is a symplectic variety and the morphism $f: Y(A, \delta) \rightarrow Y(A, 0)$ is a symplectic resolution.

Proof. The construction of $Y(A, \delta)$ and $Y(A, 0)$ as Hamiltonian reductions means that they are Poisson varieties and $f$ preserves the Poisson structure. Therefore the smooth locus of $Y(A, 0)$ is a symplectic manifold since $Y(A, \delta)$ is a symplectic manifold. In [Proudfoot and Webster 2007, §2], a stratification of $Y(A, 0)$ into smooth locally closed subvarieties of even dimensions is constructed. This stratification shows that $Y(A, 0)$ is smooth in codimension one. Therefore the fact (Lemma 4.9) that $Y(A, 0)$ is Cohen-Macaulay together with Serre's normality criterion [Bruns and Herzog 1993, Theorem 2.2.22] implies that $Y(A, 0)$ is normal. Also, the fact 
that $Y(A, \delta)$ is a symplectic manifold implies that its canonical bundle is trivial. Therefore the Grauert-Riemenschneider vanishing theorem implies that $Y(A, 0)$ has rational Gorenstein singularities. Then [Namikawa 2001, Theorem 6] says that $Y(A, 0)$ is a symplectic variety.

4H. G.I.T. chambers for hypertoric varieties. Define the subvariety $\mathscr{E}$ of $T^{*} V$ by $\mathscr{E}=\left\{(x, y) \in T^{*} V \mid x_{i} \cdot y_{i}=0\right.$ for all $\left.i \in[1, n]\right\}$. We decompose $\mathscr{E}$ into its $n$-dimensional irreducible components

$\mathscr{E}=\bigcup_{I \subset[1, n]} \mathscr{E}_{I}, \quad \mathscr{E}_{I}:=\left\{(x, y) \in T^{*} V \mid x_{i}=0\right.$ for all $i \in I, y_{i}=0$ for all $\left.i \in[1, n] \backslash I\right\}$.

The subvariety $\mathscr{E}$ is preserved under the $\mathbb{T}$-action. Therefore we may consider the corresponding G.I.T. quotients. The G.I.T. quotient $\mathscr{E} / / \delta \mathbb{\mathbb { T }}$ is a closed subvariety of $Y(A, \delta)$, called the extended core of $Y(A, \delta)$; see [Proudfoot 2008] for details.

Lemma 4.12. In $\mathbb{X}_{\mathbb{Q}}$ we have equalities of G.I.T. fans

$$
\Delta\left(\mathbb{T}, T^{*} V\right)=\Delta(\mathbb{T}, \mathscr{E})=\Delta\left(\mathbb{T}, \mu^{-1}(0)\right) .
$$

Proof. For a fixed $I \subset[1, n]$, denote by $\pi_{I}: T^{*} V \rightarrow \mathscr{E}_{I}$ the projection that sends $x_{i}$ to zero if $i \in I$ and $y_{j}$ to zero if $j \in[1, n] \backslash I$. The restriction of $\pi_{I}$ to $\mu^{-1}(0)$ will be denoted $\tilde{\pi}_{I}$. The statement of lemma follows from the claim

$$
\left(T^{*} V\right)_{\delta}^{\mathrm{ss}}=\bigcup_{I \subset[1, n]} \pi_{I}^{-1}\left(\left(\mathscr{E}_{I}\right)_{\delta}^{\mathrm{ss}}\right) \quad \text { and } \quad\left(\mu^{-1}(0)\right)_{\delta}^{\mathrm{ss}}=\bigcup_{I \subset[1, n]}\left(\tilde{\pi}_{I}\right)^{-1}\left(\left(\mathscr{E}_{I}\right)_{\delta}^{\mathrm{ss}}\right)
$$

for each $\delta \in \mathbb{X}$. Let $p \in\left(\mathscr{E}_{I}\right)_{\delta}^{\mathrm{ss}}$. Then, without loss of generality, we may assume that there exists a monomial $f \in R^{N \delta}, N \geq 1$, such that $f(p) \neq 0$. Then $f(q) \neq 0$ for all $q \in \pi_{I}^{-1}(p)$. Hence $\left(T^{*} V\right)_{\delta}^{\mathrm{ss}} \supset \pi_{I}^{-1}\left(\left(\mathscr{E}_{I}\right)_{\delta}^{\mathrm{ss}}\right)$ and $\left(\mu^{-1}(0)\right)_{\delta}^{\mathrm{ss}} \supset\left(\tilde{\pi}_{I}\right)^{-1}\left(\left(\mathscr{E}_{I}\right)_{\delta}^{\mathrm{ss}}\right)$ for all $I \subset[1, n]$. Now choose $p \in\left(T^{*} V\right)_{\delta}^{\mathrm{ss}}$. Then there exist $m \in \mathbb{N}$ and $g \in R^{m \delta}$ such that $g(p) \neq 0$. We may assume without loss of generality that

$$
g=\prod_{i} x_{i}^{u_{i}} \prod_{i} y_{i}^{v_{i}} \quad \text { for some } u_{i}, v_{i} \geq 0 .
$$

By definition, $\sum_{i}\left(u_{i}-v_{i}\right) a_{i}=m \delta$. For each $i$, define $s_{i}$ and $t_{i}$ by

(1) $u_{i}-v_{i}>0 \Longrightarrow s_{i}=u_{i}-v_{i}, t_{i}=0$;

(2) $u_{i}-v_{i}<0 \Longrightarrow t_{i}=v_{i}-u_{i}, s_{i}=0$

(3) $u_{i}-v_{i}=0 \Longrightarrow s_{i}=t_{i}=0$.

Set $I=\left\{i \in[1, n] \mid t_{i} \neq 0\right\}$. Then $g(p) \neq 0$ implies that $\pi_{I}(p) \neq 0$. Define $\tilde{g}=\prod_{i} x_{i}^{s_{i}} \prod_{i} y_{i}^{t_{i}} \in R^{m \delta}$. Then $\tilde{g}\left(\pi_{I}(p)\right) \neq 0$ implies that $p \in \pi_{I}^{-1}\left(\left(\mathscr{E}_{I}\right)_{\delta}^{\mathrm{ss}}\right)$ and hence $\left(T^{*} V\right)_{\delta}^{\mathrm{ss}}=\bigcup_{I \subset[1, n]} \pi_{I}^{-1}\left(\left(\mathscr{E}_{I}\right)_{\delta}^{\mathrm{ss}}\right)$ as required. The second equality in (13) follows from the first one. 
Corollary 4.13. Let $\mathbb{T}$ act on $V$ via $A$ as in Section $4 B$. Then $\left|\Delta\left(\mathbb{T}, \mu^{-1}(0)\right)\right|=\mathbb{X}_{\mathbb{Q}}$ and the walls of the fan $\Delta\left(\mathbb{T}, \mu^{-1}(0)\right)$ are $\sum_{i \in J} \mathbb{Q} \cdot a_{i}$, where $J \subset[1, n]$ is any subset such that $\operatorname{dim}_{\mathbb{Q}}\left(\operatorname{Span}_{\mathbb{Q}}\left(a_{i} \mid i \in J\right)\right)=d-1$.

Assume now that $A$ is unimodular and choose $\delta \in \mathbb{X}$ to lie in the interior, denoted $C(\delta)$, of a $d$-cone of $\Delta\left(\mathbb{T}, \mu^{-1}(0)\right)$. If $\zeta \in C(\delta) \cap \mathbb{X}$ then $Y(A, \delta)=Y(A, \zeta)$. Recall from Section 3A that $\zeta$ also defines a line bundle $L_{\zeta}$ on $Y(A, \delta)$. From the definition of $Y(A, \delta)$ as proj of a graded ring, we see that $L_{\zeta}$ is an ample line bundle on $Y(A, \delta)$. Summarizing:

Lemma 4.14. Let $A$ be unimodular and let $C(\delta)$ denote the interior of a $d$-cone of $\Delta\left(\mathbb{T}, \mu^{-1}(0)\right)$. Then the line bundle $L_{\zeta}$ on $Y(A, \delta)$ is ample for all $\zeta \in C(\delta) \cap \mathbb{X}$.

\section{Quantum Hamiltonian reduction}

5A. Recall that $\mathfrak{D}(V)$ denotes the ring of algebraic differential operators on the $n$-dimensional space $V$. Let $\mathbb{T}$ act on $V$ with weights described by the matrix $A$ (as in Sections 4B and 4C) and choose an element $\chi$ of the dual $t^{*}$ of the Lie algebra $\mathfrak{t}$ of $\mathbb{T}$. As explained in Section 3D, by differentiating the action of $\mathbb{T}$ we get a quantum moment map $\mu_{D}: \mathfrak{t} \rightarrow \mathfrak{D}(V), t_{i} \mapsto \sum_{j=1}^{n} a_{i j} x_{j} \partial_{j}$. As in Section 3C, the quantum Hamiltonian reduction of $V$ with respect to $\chi$ is defined to be the noncommutative algebra

$$
\mathrm{U}_{\chi}:=\left(\mathfrak{D}(V) / \mathfrak{D}(V)\left(\mu_{D}-\chi\right)(\mathfrak{t})\right)^{\mathbb{T}} .
$$

We also have bimodules

$$
\mathrm{U}_{\chi}^{\theta}:=\left(\mathfrak{D}(V) / \mathfrak{D}(V)\left(\mu_{D}-(\chi+\theta)\right)(\mathfrak{t})\right)^{-\theta} .
$$

We say that $\chi$ and $\chi+\theta$ are comparable if the multiplication map $U_{\chi}^{\theta} \otimes U_{\chi+\theta}^{-\theta} \rightarrow U_{\chi}$ is nonzero. By [Musson and Van den Bergh 1998, Theorem 7.3.1], the ring $U_{\chi}$ is a domain. Then [ibid., Proposition 4.4.2] says that this implies that comparability is an equivalence relation. As in Section 3C, write $\chi \rightarrow \chi+\theta$ if $\mathrm{U}_{\chi+\theta}^{-\theta} \otimes \mathrm{U}_{\chi}^{\theta} \rightarrow \mathrm{U}_{\chi+\theta}$. As noted in [ibid., Remark 4.4.3], the relation $\rightarrow$ is transitive. Therefore it defines a preorder on the set of elements in $\mathfrak{t}^{*}$ comparable to $\chi$. We say that $\chi$ is maximal if $\chi$ is maximal in this preordering, that is, $\chi^{\prime} \rightarrow \chi$ implies $\chi \rightarrow \chi^{\prime}$.

5B. The main results. Write pr: $\mathbb{C} \rightarrow \mathbb{Q}$ for the $\mathbb{Q}$-linear projection onto $\mathbb{Q}$ and denote by the same symbol the corresponding extension to $\mathfrak{t}^{*}$ :

$$
\text { pr: } \mathfrak{t}^{*}=\mathbb{X} \otimes_{\mathbb{Z}} \mathbb{C} \longrightarrow \mathbb{X}_{\mathbb{Q}} .
$$

We also write pr for the map $\mathbb{C}^{n}=\mathbb{C} \otimes_{\mathbb{Z}} \mathbb{Z}^{n} \rightarrow \mathbb{Q}^{n}$. Then $\operatorname{pr}(A \cdot v)=A \cdot \operatorname{pr}(v)$ for all $v \in \mathbb{C}^{n}$. The following proposition is the key to proving our main result. Its proof is given in Section 5D. 
Proposition 5.1. Let $C \subset \mathbb{X}_{\mathbb{Q}}$ be the interior of a $d$-cone in the fan $\Delta\left(\mathbb{T}, \mu^{-1}(0)\right)$. Choose $\chi \in \mathfrak{t}^{*}$ such that $\operatorname{pr}(\chi) \in C$. Then there exists a nonempty $d$-dimensional integral cone $C(\chi) \subset C \cap \mathbb{X} \cup\{0\}$ such that for all $\theta \in C(\chi), \chi \leftarrow \chi+p \theta$ for all $p \in \mathbb{Z}_{\geq 0}$.

Recall from Sections 4F and 3D that for each $\chi \in \mathfrak{t}^{*}$ and $\delta \in C$, where $C$ is the interior of a $d$-cone of $\Delta\left(\mathbb{T}, \mu^{-1}(0)\right)$, we have defined the sheaf of algebras $\mathscr{A}_{\chi}$ on the smooth symplectic manifold $Y(A, \delta)$.

Theorem 5.2. Let $C \subset \mathbb{X}_{\mathbb{Q}}$ be the interior of a d-cone of $\Delta\left(\mathbb{T}, \mu^{-1}(0)\right)$. Choose $\chi \in \mathfrak{t}^{*}$ such that $\operatorname{pr}(\chi) \in C$ and choose $\delta \in C$. Let $\mathscr{A}_{\chi}$ be the corresponding $W$-algebra on $Y(A, \delta)$.

(i) The functor $\operatorname{Hom}_{\operatorname{Mod}_{F}^{\text {good }}\left(\mathscr{A}_{\chi}\right)}\left(\mathscr{A}_{\chi}, \cdot\right)$ defines an equivalence of categories

$$
\underline{\operatorname{Mod}}_{F}^{\text {good }}\left(\mathscr{A}_{\chi}\right) \stackrel{\sim}{\longrightarrow} \mathrm{U}_{\chi}-\bmod
$$

with quasiinverse $\mathscr{A}_{\chi} \otimes \mathrm{U}_{\chi}(\cdot)$.

(ii) For any $0 \neq \theta \in C(\chi)$, there exists some $N>0$ such that the functor $\operatorname{Hom}_{\operatorname{Mod}_{F}^{\mathrm{good}}\left(\mathscr{A}_{\chi}\right)}\left(\mathscr{A}_{\chi}, \cdot\right)$ defines an equivalence of categories

$$
\operatorname{Mod}_{F}^{\text {good }}\left(\mathscr{A}_{\chi}+N \theta\right) \stackrel{\sim}{\longrightarrow} \mathrm{U}_{\chi+N \theta}-\bmod
$$

with quasiinverse $\mathscr{A}_{\chi} \otimes_{\mathrm{U}_{\chi}}(\cdot)$.

Proof. By Proposition 5.1 we can choose $0 \neq \theta \in C(\chi)$ such that $\chi \leftarrow \chi+p \theta$ for all $p \in \mathbb{Z}_{\geq 0}$. Since $C(\chi) \backslash\{0\} \subset C$, Lemma 4.14 says that $\theta$ defines an ample line bundle $L_{\theta}$ on $Y(A, \delta)$ and we have $\mathscr{A}_{\chi, \theta}(0) / \mathscr{A}_{\chi, \theta}(-1 / 2) \simeq L_{-\theta}$. Then part (i) of the theorem is a particular case of Theorem 3.3 (ii). The proof of Proposition 5.1 shows that we actually have

$$
\chi \leftarrow \chi+\theta \leftarrow \chi+2 \theta \leftarrow \cdots .
$$

Since the set of all covectors of the oriented matroid defined by $A$ is finite and each $2_{\chi}$ (which will be defined in Definition 5.9) is a subset of this set, we see that there are only finitely many different $2_{\chi}$. Therefore we eventually get

$$
\chi+N \theta \leftrightarrows \chi+(N+1) \theta \leftrightarrows \cdots
$$

for some sufficiently large $N$. Then part (ii) of the theorem is a particular case of Theorem 3.3(iii).

Corollary 5.3. Let $Y(A, \delta), A_{\chi}, \mathrm{U}_{\chi}, \ldots$ be as in Theorem 5.2 (with $\left.\operatorname{pr}(\chi) \in C\right)$. If the global dimension of $\mathrm{U}_{\chi}$ is finite then the functor $\operatorname{Hom}_{\operatorname{Mod}_{F}^{\text {good }}\left(\mathscr{A}_{\chi}\right)}\left(\mathscr{A}_{\chi}, \cdot\right)$ defines an equivalence of categories $\operatorname{Mod}_{F}^{\mathrm{good}}\left(\mathscr{A}_{\chi}\right) \stackrel{\sim}{\longrightarrow} \mathrm{U}_{\chi}$-mod with quasiinverse $A_{\chi} \otimes u_{\chi}(\cdot)$. 
Proof. By Proposition 5.1 we can choose $0 \neq \theta \in C(\chi)$ such that $\chi \leftarrow \chi+p \theta$ for all $p \in \mathbb{Z}_{\geq 0}$. However, [Musson and Van den Bergh 1998, Theorem 9.1.1] says that $\chi$ is maximal if and only if the global dimension of $\mathrm{U}_{\chi}$ is finite. Therefore $\chi \leftrightarrows \chi+\theta$ for all $\theta \in C(\chi)$. Then, as in the proof of Theorem 5.2, Theorem 3.3 implies the statement of the corollary.

It seems natural to conjecture that, for any $\chi \in \mathfrak{t}^{*}, \mathrm{U}_{\chi}$ has finite global dimension if and only if $\operatorname{Mod}_{F}^{\text {good }}\left(\mathscr{A}_{\chi}\right) \stackrel{\sim}{\longrightarrow} \mathrm{U}_{\chi}$-mod, where $\mathscr{A}_{\chi}$ is the corresponding $W$-algebra, defined on some $Y(A, \delta)$.

5C. The case $\boldsymbol{d}=1$. In specific cases it is possible to strengthen Proposition 5.1. One such case is when the torus $\mathbb{T}$ is one-dimensional. Here the sets $\mathscr{2}_{\chi}$ (which will be defined in Definition 5.9) can be explicitly described, as was done in [Van den Bergh 1991]. Since $A$ is assumed to be unimodular and $a_{i} \neq 0$ for all $i$ we see that $a_{i}= \pm 1$ for all $i$. After reordering we may assume that $a_{1}, \ldots, a_{k}=1$ and $a_{k+1}, \ldots, a_{n}=-1$. For simplicity let us assume that $n>1$. Then

$$
\begin{aligned}
& \mathscr{2}_{\chi}=\{0\} \quad \Longleftrightarrow \chi \in(\mathbb{C} \backslash \mathbb{Z}) \cup\{k-n+1, k-n+2, \ldots, n-k-2, n-k-1\}, \\
& \mathscr{2}_{\chi}=\{0,+\} \Longleftrightarrow \chi \in \mathbb{Z}_{\geq n-k}, \\
& \mathscr{2}_{\chi}=\{0,-\} \Longleftrightarrow \chi \in \mathbb{Z}_{\leq k-n} .
\end{aligned}
$$

In this situation $\mathbb{X}_{\mathbb{Q}}=\mathbb{Q}$ and there are two 1-cones with respect to the action of $\mathbb{T}$ on $\mu^{-1}(0)$; they are $\mathbb{Q}_{\geq 0}$ and $\mathbb{Q}_{\leq 0}$. Applying Theorem 3.3 gives:

Proposition 5.4. Let $\operatorname{dim} \mathbb{T}=1$ and $n>1$ and choose $\chi \in \mathfrak{t}^{*}$. For $\delta \neq 0$, let $\mathscr{A}_{\chi}$ denote the corresponding $W$-algebra on $Y(A, \delta)$.

(i) When $\delta=1$ we have an equivalence

$$
\underline{\operatorname{Mod}}_{F}^{\text {good }}\left(\mathscr{A}_{\chi}\right) \stackrel{\sim}{\longrightarrow} \mathrm{U}_{\chi}-\bmod
$$

if and only if $\chi \in(\mathbb{C} \backslash \mathbb{Z}) \cup \mathbb{Z}_{\geq 0}$ and $\underline{\operatorname{Mod}}_{F}^{\text {good }}\left(\mathscr{A}_{\chi}\right)=\operatorname{Mod}_{F}^{\text {good }}\left(\mathscr{A}_{\chi}\right)$ if and only if $\chi \in(\mathbb{C} \backslash \mathbb{Z}) \cup \mathbb{Z}_{\geq n-k}$.

(ii) When $\delta=-1$ we have an equivalence $\operatorname{Mod}_{F}^{\text {good }}\left(\mathscr{A}_{\chi}\right) \stackrel{\sim}{\longrightarrow} \mathrm{U}_{\chi}$-mod if and only if $\chi \in(\mathbb{C} \backslash \mathbb{Z}) \cup \mathbb{Z}_{<0}$ and $\underline{\operatorname{Mod}}_{F}^{\text {good }}\left(\mathscr{A}_{\chi}\right)=\operatorname{Mod}_{F}^{\text {good }}\left(\mathscr{A}_{\chi}\right)$ if and only if $\chi \in(\mathbb{C} \backslash \mathbb{Z}) \cup \mathbb{Z}_{\leq k-n}$.

This result can be viewed as a variant of [Van den Bergh 1991, Theorem 6.1.3], where sufficient conditions for the $D$-affinity of weighted projective spaces are stated.

5D. The remainder of this section is devoted to the proof of Proposition 5.1. Since $\mathbb{T}$ can be considered as a subgroup of $\mathbb{T}^{n}, \mathfrak{t}$ is a Lie subalgebra of $\mathfrak{g}=\operatorname{Lie}\left(\mathbb{T}^{n}\right)$ and we may regard elements of $\mathfrak{t}$ as linear functionals on $\mathfrak{g}^{*}$. Let $\rho: \mathfrak{g}^{*} \rightarrow \mathfrak{t}^{*}$ be the natural map. 
Definition 5.5. Let $\lambda \in \mathbb{Y}$ and $\theta \in\left(\sum_{\left\langle\lambda, a_{i}\right\rangle=0} \mathbb{C} \cdot a_{i}\right) /\left(\sum_{\left\langle\lambda, a_{i}\right\rangle=0} \mathbb{Z} \cdot a_{i}\right)$. We say that the pair $(\lambda, \theta)$ is attached to $\chi$ if there exists $\alpha \in \rho^{-1}(\chi)$ such that

$$
\sum_{\left\langle\lambda, a_{i}\right\rangle=0} \alpha_{i} a_{i} \equiv \theta \quad \bmod \sum_{\left\langle\lambda, a_{i}\right\rangle=0} \mathbb{Z} \cdot a_{i}
$$

and

$$
\begin{aligned}
& \left\langle\lambda, a_{i}\right\rangle>0 \Longrightarrow \alpha_{i} \in \mathbb{Z}, \alpha_{i} \geq 0, \\
& \left\langle\lambda, a_{i}\right\rangle<0 \Longrightarrow \alpha_{i} \in \mathbb{Z}, \alpha_{i}<0, \\
& \left\langle\lambda, a_{i}\right\rangle=0 \Longrightarrow \alpha_{i} \in \mathbb{C} \backslash \mathbb{Z} .
\end{aligned}
$$

Remark 5.6. The above definition is based on [Musson and Van den Bergh 1998, Definition 7.2.1]. There it is stipulated that $\lambda \in \mathfrak{t} \cap \mathbb{Q}^{n}$, but we only care about whether $\left\langle\lambda, a_{i}\right\rangle$ is greater than, less than or equal to 0 ; therefore we can assume $\lambda \in \mathbb{Y}$. Also our sign convention in Definition 5.5 is opposite to that given in [ibid., Definition 7.2.1] so that it agrees with the conventions of Section 4.

5E. Let us define an equivalence relation on the set of pairs $(\lambda, \theta)$ by saying that $\left(\lambda_{1}, \theta_{1}\right)$ is equivalent to $\left(\lambda_{2}, \theta_{2}\right)$ if $\left\{i \mid\left\langle\lambda_{1}, a_{i}\right\rangle>0\right\}=\left\{i \mid\left\langle\lambda_{2}, a_{i}\right\rangle>0\right\}$, $\left\{i \mid\left\langle\lambda_{1}, a_{i}\right\rangle<0\right\}=\left\{i \mid\left\langle\lambda_{2}, a_{i}\right\rangle<0\right\}$ and $\theta_{2} \equiv \theta_{1} \bmod \sum_{\left\langle\lambda_{1}, a_{i}\right\rangle=0} \mathbb{Z} \cdot a_{i}$. Denote by $\mathscr{P}_{\chi}$ the set of equivalence classes of pairs $(\lambda, \theta)$ that are attached to $\chi$. The set of all possible $\lambda$ up to equivalence consist of the (finitely many) covectors of the oriented matroid defined by $A$. It will be convenient to parametrize each $\lambda$ (again up to equivalence) as an element in $\{+, 0,-\}^{n}, \lambda \leftrightarrow\left(e_{i}\right)_{i \in[1, n]}$ with $e_{i}=+$ if $\left\langle\lambda, a_{i}\right\rangle>0$ and so forth. Note, however, that not every element of $\{+, 0,-\}^{n}$ can be realized as some $\lambda$.

Proposition 5.7 [Musson and Van den Bergh 1998, Proposition 7.7.1]. Choose $\chi, \chi^{\prime} \in \mathfrak{t}^{*}$. Then, the set $\mathscr{P}_{\chi}$ parametrizes the primitive ideals in $\mathrm{U}_{\chi}$ and $\chi \rightarrow \chi^{\prime}$ if and only if $\mathscr{P}_{\chi^{\prime}} \subseteq \mathscr{P}_{\chi}$.

Since we are interested in sheaves of $W$-algebras on smooth hypertoric varieties we may assume that $A$ is unimodular. This allows us to remove $\theta$ from the description of $\mathscr{P}_{\chi}$.

Lemma 5.8. Assume that $A$ is unimodular and let $(\lambda, \theta)$ and $(\lambda, \vartheta)$, be attached to $\chi$ via $\alpha \in \rho^{-1}(\chi)$ and $\beta \in \rho^{-1}(\chi)$, respectively. Then $(\lambda, \theta)$ is equivalent to $(\lambda, \vartheta)$.

Proof. By definition, $\theta$ is the equivalence class of $\sum_{\left\langle\lambda, a_{i}\right\rangle=0} \alpha_{i} a_{i}$ in the quotient $\left(\sum_{\left\langle\lambda, a_{i}\right\rangle=0} \mathbb{C} \cdot a_{i}\right) /\left(\sum_{\left\langle\lambda, a_{i}\right\rangle=0} \mathbb{Z} \cdot a_{i}\right)$, and similarly for $\vartheta$. Therefore we must show that $\sum_{i=1}^{n} \alpha_{i} a_{i}=\sum_{i=1}^{n} \beta_{i} a_{i}$ implies

$$
\sum_{\left\langle\lambda, a_{i}\right\rangle=0}^{n} \alpha_{i} a_{i} \equiv \sum_{\left\langle\lambda, a_{i}\right\rangle=0}^{n} \beta_{i} a_{i} \bmod \sum_{\left\langle\lambda, a_{i}\right\rangle=0} \mathbb{Z} \cdot a_{i} .
$$


Choose $\left\{a_{i_{1}}, \ldots, a_{i_{k}}\right\} \subset\left\{a_{i} \mid\left\langle\lambda, a_{i}\right\rangle=0\right\}$ to be a basis of the space spanned by the set $\left\{a_{i} \mid\left\langle\lambda, a_{i}\right\rangle=0\right\}$. We can extend this to a basis $a_{i_{1}}, \ldots, a_{i_{k}}, a_{i_{k+1}}, \ldots, a_{d}$ of $\mathfrak{t}^{*}$. Since $A$ is unimodular the determinant of this basis is \pm 1 . Hence $\left\{a_{i_{1}}, \ldots, a_{i_{k}}\right\}$ span a direct summand of the lattice $\mathbb{X}$. This implies

$$
\left(\sum_{\left\langle\lambda, a_{i}\right\rangle=0} \mathbb{C} \cdot a_{i}\right) \cap \mathbb{X}=\sum_{\left\langle\lambda, a_{i}\right\rangle=0} \mathbb{Z} \cdot a_{i},
$$

which in turn implies (14).

It is shown in [ibid., Example 7.2.7] that $\theta$ is not defined up to equivalence by $\chi$ and $\lambda$ if $A$ is not unimodular.

5F. Based on Lemma 5.8, we make the following definition.

Definition 5.9. Let $\lambda \in \mathbb{Y}$ and $\chi \in \mathfrak{t}^{*}$. We say that $\lambda$ is attached to $\chi$ if there exists $\alpha \in \rho^{-1}(\chi)$ such that

$$
\begin{aligned}
& \left\langle\lambda, a_{i}\right\rangle>0 \Longrightarrow \alpha_{i} \in \mathbb{Z}, \alpha_{i} \geq 0, \\
& \left\langle\lambda, a_{i}\right\rangle<0 \Longrightarrow \alpha_{i} \in \mathbb{Z}, \alpha_{i}<0, \\
& \left\langle\lambda, a_{i}\right\rangle=0 \Longrightarrow \alpha_{i} \in \mathbb{C} \backslash \mathbb{Z} .
\end{aligned}
$$

If $\lambda_{1}, \lambda_{2} \in \mathbb{Y}$ are attached to $\chi$ then we say that $\lambda_{1}$ is equivalent to $\lambda_{2}$ if

$$
\left\{i \mid\left\langle\lambda_{1}, a_{i}\right\rangle>0\right\}=\left\{i \mid\left\langle\lambda_{2}, a_{i}\right\rangle>0\right\} \quad \text { and } \quad\left\{i \mid\left\langle\lambda_{1}, a_{i}\right\rangle<0\right\}=\left\{i \mid\left\langle\lambda_{2}, a_{i}\right\rangle<0\right\} .
$$

Let $2_{\chi}$ denote the set of equivalence classes of elements in $\mathbb{Y}$ that are attached to $\chi$.

Lemma 5.10. Assume that $A$ is unimodular. Then $\chi \rightarrow \chi^{\prime}$ if and only if $2_{\chi^{\prime}} \subseteq 2_{\chi}$ and $\chi-\chi^{\prime} \in \mathbb{X}$.

Proof. If $\chi \rightarrow \chi^{\prime}$ then clearly $\chi-\chi^{\prime} \in \mathbb{X}$ and Proposition 5.7 implies that $\mathscr{P}_{\chi^{\prime}} \subseteq \mathscr{P}_{\chi}$. This implies that $2_{\chi^{\prime}} \subseteq 2_{\chi}$.

Now assume that $2_{\chi^{\prime}} \subseteq 2_{\chi}$ and $\chi-\chi^{\prime} \in \mathbb{X}$. Let $\lambda \in 2_{\chi^{\prime}}$ and choose $\alpha \in \rho^{-1}\left(\chi^{\prime}\right)$, respectively $\beta \in \rho^{-1}(\chi)$, satisfying the conditions of Definition 5.9 for $\lambda$ with respect to $\chi^{\prime}$, respectively $\chi$. Write $\alpha=\alpha^{(1)}+\alpha^{(2)}$, where $\left(\alpha^{(1)}\right)_{i}=\alpha_{i}$ if $\left\langle\lambda, a_{i}\right\rangle \neq 0$ and $\left(\alpha^{(1)}\right)_{i}=0$ if $\left\langle\lambda, a_{i}\right\rangle=0$. Decompose $\beta=\beta^{(1)}+\beta^{(2)}$ in a similar fashion. Then

$$
\left(\chi-\chi^{\prime}\right)-\rho\left(\beta^{(1)}-\alpha^{(1)}\right) \in\left(\sum_{\left\langle\lambda, a_{i}\right\rangle=0} \mathbb{C} \cdot a_{i}\right) \cap \mathbb{X},
$$

which, by (15), equals $\sum_{\left\langle\lambda, a_{i}\right\rangle=0} \mathbb{Z} \cdot a_{i}$. Therefore we can choose $u \in \mathbb{Z}^{n}$ such that $u_{i}=0$ for all $i$ such that $\left\langle\lambda, a_{i}\right\rangle=0$ and $\rho(u)=\left(\chi-\chi^{\prime}\right)-\rho\left(\beta^{(1)}-\alpha^{(1)}\right)$. Define $\delta^{(2)}=\alpha^{(2)}+u$ and $\delta=\beta^{(1)}+\delta^{(2)}$ so that $\rho(\delta)=\chi$. We have

$$
\bar{\delta}^{(2)}=\bar{\alpha}^{(2)} \in\left(\sum_{\left\langle\lambda, a_{i}\right\rangle=0} \mathbb{C} \cdot a_{i}\right) /\left(\sum_{\left\langle\lambda, a_{i}\right\rangle=0} \mathbb{Z} \cdot a_{i}\right)
$$


and $\left(\lambda, \bar{\alpha}^{(2)}\right)$ and $\left(\lambda, \bar{\delta}^{(2)}\right)$ are attached to $\chi^{\prime}$ and $\chi$, respectively, in the sense of Definition 5.5. Therefore $\left(\lambda, \bar{\delta}^{(2)}\right)=\left(\lambda, \bar{\alpha}^{(2)}\right) \in \mathscr{P}_{\chi}$ implies that $\mathscr{P}_{\chi^{\prime}} \subseteq \mathscr{P}_{\chi}$. Hence Proposition 5.7 implies that $\chi \rightarrow \chi^{\prime}$.

Proof of Proposition 5.1. As was stated in Section 4D, the cone $\bar{C}$ is a rational cone. Therefore we can choose $\mu_{1}, \ldots, \mu_{k}$ in $\mathbb{Y}$ such that

$$
\bar{C}=\left\{\chi \in \mathbb{X}_{\mathbb{R}} \mid\left\langle\mu_{i}, \chi\right\rangle \geq 0 \text { for all } i \in[1, k]\right\}
$$

$$
\supset\left\{\chi \in \mathbb{X}_{\mathbb{R}} \mid\left\langle\mu_{i}, \chi\right\rangle>0 \text { for all } i \in[1, k]\right\}=C .
$$

We will construct $C(\chi)$ in three stages.

Claim 1. There exists an integer $N_{0} \gg 0$ such that $p N_{0} \cdot \operatorname{pr}(\chi) \in \mathbb{X} \cap C$ and $\chi+p N_{0} \cdot \operatorname{pr}(\chi) \rightarrow \chi$ for all $p \in \mathbb{N}$.

For each $\lambda \in 2_{\chi}$ fix an element $\beta^{\lambda} \in \rho^{-1}(\chi)$ such that $\beta^{\lambda}$ satisfies the properties listed in Definition 5.9 with respect to $\lambda$. Then $\operatorname{pr}(\chi)=\sum_{i=1}^{n} \operatorname{pr}\left(\beta_{i}^{\lambda}\right) a_{i}$ and we choose $N_{0}$ such that $N_{0} \cdot \operatorname{pr}\left(\beta_{i}^{\lambda}\right) \in \mathbb{Z}$ for all $\lambda \in 2_{\chi}$ and all $i$. The element $\left(\beta_{i}^{\lambda}+p N_{0} \beta_{i}^{\lambda}\right)_{i \in[1, n]}$ in $\mathfrak{g}^{*}$ satisfies the properties of Definition 5.9 with respect to $\lambda$ hence $2_{\chi} \subseteq 2_{\chi}+p N_{0} \cdot \operatorname{pr}(\chi)$. Since $p N_{0} \cdot \operatorname{pr}(\chi) \in \mathbb{X}$, Lemma 5.10 says that $\chi+p N_{0} \cdot \operatorname{pr}(\chi) \rightarrow \chi$ for all $p \in \mathbb{N}$. Note also that

$$
\left\langle\mu_{i}, \operatorname{pr}\left(\chi+p N_{0} \cdot \operatorname{pr}(\chi)\right)\right\rangle=\left(1+p N_{0}\right)\left\langle\mu_{i}, \operatorname{pr}(\chi)\right\rangle>0
$$

for all $i$ shows that $\operatorname{pr}\left(\chi+p N_{0} \cdot \operatorname{pr}(\chi)\right) \in C$.

Claim 2. Fix $\delta=\sum_{i=1}^{n} a_{i} \in \mathbb{X}$. There exists an integer $N_{1} \gg 0$ such that

$$
N_{1} \cdot \operatorname{pr}(\chi)+\delta \in \mathbb{X} \cap C \quad \text { and } \quad \chi+p\left(N_{1} \cdot \operatorname{pr}(\chi)+\delta\right) \rightarrow \chi
$$

for all $p \in \mathbb{N}$. Moreover, for all $\lambda \in \mathscr{P}_{\chi}$, there exists $\beta^{\lambda}$ as before except that $\beta_{i}^{\lambda} \neq 0$ for all $i$.

Choose $N_{1}=p N_{0}$ such that

$$
\left(N_{1} / d\right) \cdot\left\langle\mu_{i}, \operatorname{pr}(\chi)\right\rangle>\left|\left\langle\mu_{i}, a_{j}\right\rangle\right|
$$

for all $i \in[1, k]$ and $j \in[1, n]$. Let $\beta^{\lambda} \in \rho^{-1}\left(\chi+N_{1} \cdot \operatorname{pr}(\chi)\right)$ satisfy Definition 5.9 with respect to $\lambda$ for $\lambda \in 2_{\chi}$. By choosing a larger $p$ if necessary we may assume that $\beta_{i}^{\lambda} \in \mathbb{Z} \backslash\{0\}$ implies that $\left|\beta_{i}^{\lambda}\right|>1$. Then $\beta_{i}^{\lambda}+1<0$ if $\beta_{i}^{\lambda} \in \mathbb{Z}_{<0}$ and $\beta_{i}^{\lambda}+1>0$ if $\beta_{i}^{\lambda} \in \mathbb{Z}_{\geq 0}$. Moreover $\left(\beta_{i}^{\lambda}+1\right)_{i \in[1, n]}$ satisfies (16) with respect to $\lambda$,

$$
\sum_{i=1}^{n}\left(\beta_{i}^{\lambda}+1\right) a_{i}=\chi+\left(N_{1} \cdot \operatorname{pr}(\chi)+\delta\right),
$$

and hence $\chi+\left(N_{1} \cdot \operatorname{pr}(\chi)+\delta\right) \rightarrow \chi$. The same holds for all $\chi+p\left(N_{1} \cdot \operatorname{pr}(\chi)+\delta\right)$. Finally (17) implies that $\operatorname{pr}\left(\chi+q\left(N_{1} \cdot \operatorname{pr}(\chi)+\delta\right)\right) \in C$ for all $q \in \mathbb{Z}_{\geq 0}$. 
Proof of the proposition. Note that (17) implies $p\left(N_{1} \cdot \operatorname{pr}(\chi)+\delta\right) \in C$ for all $p$ as well. Let

$$
I_{\chi}=\left\{\epsilon \in(-1,1)^{n} \subset \mathbb{Q}^{n} \mid-\left\langle\mu_{i},\left(N_{1} \cdot \operatorname{pr}(\chi)+\delta\right)\right\rangle<\left\langle\mu_{i}, \epsilon\right\rangle \text { for all } i\right\} .
$$

Since $C$ is $d$-dimensional, there exists some $0<c<1$ such that $[-c, c]^{n} \subset I_{\chi}$. Let $\left\{v_{j} \mid j \in\left[1,2^{n}\right]\right\} \subset[-c, c]^{n}$ be the vertices of the box. Choose $p \in \mathbb{N}$ such that $p \cdot v_{j} \in \mathbb{Z}^{n}$ for all $j$. The same argument as in Claims 1 and 2 shows

$$
\chi \leftarrow \chi+q\left(p\left(N_{1} \cdot \operatorname{pr}(\chi)+\delta+A \cdot v_{j}\right)\right) \text { for all } q \in \mathbb{Z}_{\geq 0} .
$$

We set $u_{j}=p\left(N_{1} \cdot \operatorname{pr}(\chi)+\delta+A \cdot v_{j}\right)$. One can check as above $\chi \leftarrow \chi+\sum_{i=1}^{2^{n}} k_{i} \cdot u_{i}$ for all $k_{i} \in \mathbb{Z}_{\geq 0}$.

Remark 5.11. We conclude with a couple of remarks regarding Proposition 5.1.

(i) Note that in the proof of Proposition 5.1 we only used the fact that $C$ is the interior of some $d$-dimensional rational cone.

(ii) In general, the proposition is false when $\operatorname{pr}(\chi) \in C$ is replaced by $\operatorname{pr}(\chi) \in \bar{C}$.

(iii) It would be very interesting to directly relate the sets $2_{\chi}$ to the G.I.T. fan.

\section{The rational Cherednik algebra associated to cyclic groups}

6A. As explained in the introduction, the original motivation for this article was to reproduce the results of [Kashiwara and Rouquier 2008] for the rational Cherednik algebra $H_{\boldsymbol{h}}\left(\mathbb{Z}_{m}\right)$ associated to the cyclic group $\mathbb{Z}_{m}$. These rational Cherednik algebras are parametrized ${ }^{3}$ by an $m$-tuple $\boldsymbol{h}=\left(h_{i}\right)_{i \in[0, m-1]} \in \mathbb{C}^{m}$, where the indices are taken modulo $m$. We fix a one-dimensional space $\mathfrak{h}=\mathbb{C} \cdot y$ and $\mathfrak{h}^{*}=\mathbb{C} \cdot x$ such that $\langle x, y\rangle=1$. The cyclic group $\mathbb{Z}_{m}=\langle\varepsilon\rangle$ acts on $\mathfrak{h}$ and $\mathfrak{h}^{*}$ via $\varepsilon \cdot y=\zeta^{-1} y$ and $\varepsilon \cdot x=\zeta x$, where $\zeta$ is a fixed primitive $m$-th root of unity. The idempotents in $\mathbb{C}_{m}$ corresponding to the simple $\mathbb{Z}_{m}$-modules are

$$
e_{i}=\frac{1}{m} \sum_{j=0}^{m-1} \zeta^{-i j} \varepsilon^{j}, \quad i \in[0, m-1]
$$

so that $\varepsilon \cdot e_{i}=\zeta^{i} e_{i}$. Then $e_{i+1} \cdot x=x \cdot e_{i}$ and $e_{i-1} \cdot y=y \cdot e_{i}$. If we fix $\alpha_{\varepsilon^{i}}=\sqrt{2} \cdot x$ and $\alpha_{\varepsilon^{i}}^{\vee}=(-1 / \sqrt{2}) \cdot y$ then the commutation relations defining $H_{\boldsymbol{h}}\left(\mathbb{Z}_{m}\right)$, as stated in [Rouquier 2008], become

$$
\varepsilon \cdot x=\zeta x \cdot \varepsilon, \quad \varepsilon \cdot y=\zeta^{-1} y \cdot \varepsilon, \quad[y, x]=1+m \sum_{i=0}^{m-1}\left(h_{i+1}-h_{i}\right) e_{i},
$$

where indices are taken modulo $m$.

\footnotetext{
${ }^{3}$ In this paper the parameters $\left(h_{i}\right)$ and $\left(\chi_{i}\right)$ are used. However the paper [Kuwabara 2010] uses the parameters $\left(\kappa_{i}\right)$ and $\left(c_{i}\right)$. The different parametrizations are related by $h_{i} \leftrightarrow \kappa_{i}$ and $c_{i} \leftrightarrow \chi_{i}-\chi_{i+1}$.
} 
6B. The category $0 \subset H_{h}$-mod is defined to be the subcategory of all finitely generated $H_{\boldsymbol{h}}$-modules such that the action of $y \in \mathbb{C}\left[\mathfrak{h}^{*}\right]$ is locally nilpotent. It is a highest weight category. To each simple $\mathbb{Z}_{m}$-module $\mathbb{C} \cdot e_{i}$, one can associate a standard module in the category $\mathrm{O}$ defined by

$$
\Delta\left(e_{i}\right):=H_{\boldsymbol{h}} \otimes \mathbb{C}\left[\mathfrak{h}^{*}\right] \rtimes \mathbb{Z}_{m} \mathbb{C} \cdot e_{i},
$$

where $y \in \mathbb{C}\left[\mathfrak{h}^{*}\right]$ acts as zero on $e_{i}$. Each $\Delta\left(e_{i}\right)$ has a simple head $L\left(e_{i}\right)$ and $L\left(e_{i}\right) \not L L\left(e_{j}\right)$ for $i \neq j$. The set of simple modules $\left\{L\left(e_{i}\right)\right\}_{i \in[0, m-1]}$ is, up to isomorphism, all simple modules in $\mathrm{O}$. Fix $i \in[0, m-1]$ and let $c_{i}$ be the smallest element in $\mathbb{Z}_{\geq 1} \cup\{\infty\}$ such that $c_{i}+m h_{i+c_{i}}-m h_{i}=0$. The identity

$$
\left[y, x^{j}\right]=x^{j-1}\left(j+m \sum_{i=0}^{m-1}\left(h_{i+j}-h_{i}\right) e_{i}\right), \text { for all } j \geq 0,
$$

shows that $L\left(e_{i}\right)=\left(\mathbb{C}[x] /\left(x^{c_{i}}\right)\right) \otimes e_{i}$. Fix $e:=e_{0}$, the trivial idempotent. The algebra $e H_{h} e$ is called the spherical subalgebra of $H_{\boldsymbol{h}}$. Multiplication by $e$ defines a functor $e: H_{\boldsymbol{h}}$-mod $\rightarrow e H_{\boldsymbol{h}} e$-mod with left adjoint $H_{\boldsymbol{h}} e \otimes_{e H_{\boldsymbol{h}} e}(\cdot)$. Let $\mathscr{C} \subset \mathbb{C}^{m}$ be the union of the finitely many hyperplanes defined by the equations $j+m h_{i+j}-m h_{i}=0$, where $i \in[1, m-1]$ and $j \in[0, m-i]$.

Lemma 6.1. The functor $e: H_{\boldsymbol{h}}-\bmod \rightarrow e H_{\boldsymbol{h}} e-\bmod$ is an equivalence if and only if $\boldsymbol{h} \notin \mathscr{C}$. This implies that $\mathrm{H}_{\boldsymbol{h}}$ e has finite global dimension when $\boldsymbol{h} \notin \mathscr{C}$.

Proof. The functor $e$ will be an equivalence if and only if $H_{\boldsymbol{h}} e H_{\boldsymbol{h}}=H_{\boldsymbol{h}}$. By Ginzburg's generalized Duflo theorem [Ginzburg 2003, Theorem 2.3], $H_{\boldsymbol{h}} e H_{\boldsymbol{h}} \neq H_{\boldsymbol{h}}$ implies that there is some simple module in the category $\mathbb{O}$ that is annihilated by $e$. This happens if and only if $\boldsymbol{h} \in \mathscr{C}$. The second statement follows from the fact that $H_{\boldsymbol{h}}$ has finite global dimension.

6C. The minimal resolution of $\mathbb{C}^{2} / \mathbb{Z}_{m}$. In order to relate the spherical subalgebra of $H_{\boldsymbol{h}}$ to a $W$-algebra on the resolution of the corresponding Kleinian singularity $\mathbb{C}^{2} / \mathbb{Z}_{m}$, we must describe $e H_{\boldsymbol{h}} e$ as a quantum Hamiltonian reduction. Such an isomorphism is well known and is a particular case of a more general construction by Holland [1999]. First we describe the minimal resolution of $\mathbb{C}^{2} / \mathbb{Z}_{m}$ as a hypertoric variety. Let $Q$ be the cyclic quiver with vertices $V=\left\{v_{0}, \ldots, v_{m-1}\right\}$ and arrows $u_{i}: v_{i-1} \rightarrow v_{i}$ for $i \in[1, m]$ (where $v_{m}$ is identified with $v_{0}$ ). Let $v$ be the dimension vector with 1 at each vertex. Then the space of representations for $Q$ with dimension vector $v$ is the affine space

$$
\operatorname{Rep}(Q, v)=\left\{\left(u_{i}\right)_{i \in[1, m]} \mid u_{i} \in \mathbb{C}\right\} \simeq \mathbb{C}^{m}
$$

and we write $\mathbb{C}[\operatorname{Rep}(Q, v)]=\mathbb{C}\left[x_{1}, \ldots, x_{m}\right]$. There is an action of

$$
\mathbb{T}^{m}=\left\{\left(\lambda_{i}\right)_{i \in[1, m]} \mid \lambda_{i} \in \mathbb{C}^{\times}\right\}
$$


on $\operatorname{Rep}(Q, v)$ given by $\lambda \cdot u_{i}=\lambda_{i} \lambda_{i-1}^{-1} u_{i}$, and hence $\lambda \cdot x_{i}=\lambda_{i}^{-1} \lambda_{i-1} x_{i}$. The one-dimensional torus $\mathbb{T}$ embedded diagonally in $\mathbb{T}^{m}$ acts trivially on $\operatorname{Rep}(Q, v)$. Therefore $\mathbb{T}^{m-1}:=\mathbb{T}^{m} / \mathbb{T}$ acts on $\operatorname{Rep}(Q, v)$. The lattice of characters $\mathbb{X}\left(\mathbb{T}^{m-1}\right)$ is the sublattice of $\mathbb{X}\left(\mathbb{T}^{m}\right)=\bigoplus_{i=1}^{m} \mathbb{Z} \cdot v_{i}$ consisting of points $\phi=\sum_{i=1}^{m} \phi_{i} v_{i}$ such that $\sum_{i=1}^{m} \phi_{i}=0$. We fix the basis $\left\{w_{i}=v_{i}-v_{i+1} \mid i \in[0, m-2]\right\}$ of $\mathbb{X}\left(\mathbb{T}^{m-1}\right)$ so that $\phi=\left(\phi_{i}\right)_{i \in[1, n]}=\sum_{i=1}^{m-1} \chi_{i} w_{i}$, where $\chi_{i}=\sum_{j=1}^{i} \phi_{i}$. Then the $(m-1) \times m$ matrix encoding the action of $\mathbb{T}^{m-1}$ is given by

$$
A=\left(a_{1}, \ldots, a_{m}\right)=\left(\begin{array}{ccccc}
1 & 0 & \ldots & 0 & -1 \\
0 & 1 & & \vdots & -1 \\
\vdots & & \ddots & 0 & \vdots \\
0 & \ldots & 0 & 1 & -1
\end{array}\right)
$$

The G.I.T walls in $\mathfrak{t}^{*}$, where $\mathfrak{t}=\operatorname{Lie}\left(\mathbb{T}^{m-1}\right)$, are given by the hyperplanes $H_{i}=\left(\chi_{i}=0\right), i \in[1, m-1]$, and $H_{i j}=\left(\chi_{i}=\chi_{j}\right), i \neq j \in[1, m-1]$. Hence the $m$ cones are the connected components of the complement to this union of hyperplanes. As was shown originally in terms of hyperkähler manifolds by Kronheimer [1989] and then by Cassens and Slodowy [1998] in the algebraic setting, we have:

Proposition 6.2. Let $\delta$ belong to the interior of an $m$-cone. Then the hypertoric variety $Y(A, \delta)$ is isomorphic to the minimal resolution $\left(\mathbb{C}^{2} / \mathbb{Z}_{m}\right)^{\sim}$ of the Kleinian singularity $\mathbb{C}^{2} / \mathbb{Z}_{m}$.

As is well-known, the hypertoric variety $Y(A, \delta)$ is a toric variety. It is shown in [Hausel and Sturmfels 2002, Theorem 10.1] that a hypertoric variety is toric if and only if it is a product of varieties of the form $\left(\mathbb{C}^{2} / \mathbb{Z}_{m}\right)^{\sim}$ for various $m$. Let us now consider the corresponding quantum Hamiltonian reduction

$$
\mathrm{U}_{\chi}=\left(\mathfrak{D}(\operatorname{Rep}(Q, v)) / \mathfrak{D}(\operatorname{Rep}(Q, v))\left(\mu_{D}-\chi\right)(\mathfrak{t})\right)^{\mathbb{\Psi}^{m-1}} .
$$

The quantum moment map in this case is given by

$$
\mu_{D}: \mathfrak{t} \longrightarrow \mathfrak{D}(\operatorname{Rep}(Q, v)), \quad t_{i} \mapsto x_{i} \partial_{i}-x_{m} \partial_{m} \quad \text { for all } i \in[1, m-1] .
$$

Since

$$
\begin{aligned}
\mathfrak{D}(\operatorname{Rep}(Q, v))^{\mathbb{\varpi}^{m-1}} & =\left\langle\partial_{1} \cdots \partial_{m}, x_{1} \cdots x_{m}, x_{1} \partial_{1}, \ldots, x_{m} \partial_{m}\right\rangle, \\
\left\langle\left(\mu_{D}-\chi\right)(\mathfrak{t})\right\rangle & =\left\langle x_{i} \partial_{i}-x_{m} \partial_{m}-\chi_{i} \mid i \in[1, m-1]\right\rangle,
\end{aligned}
$$

where we set $\partial_{i}:=\partial / \partial x_{i}, \mathrm{U}_{\chi}$ is generated by $\partial_{1} \cdots \partial_{m}, x_{1} \cdots x_{m}$ and $x_{m} \partial_{m}$.

6D. The Dunkl embedding. Let $\mathfrak{h}_{\text {reg }}:=\mathfrak{h} \backslash\{0\}$ and denote by $\mathfrak{D}\left(\mathfrak{h}_{\text {reg }}\right)$ the ring of algebraic differential operators on $\mathfrak{h}_{\text {reg }}$. In order to show that the spherical subalgebra of $H_{\boldsymbol{h}}$ is isomorphic to a suitable quantum Hamiltonian reduction, we realize $e H_{\boldsymbol{h}} e$ as a subalgebra of $\mathfrak{D}\left(\mathfrak{h}_{\text {reg }}\right)$ using the Dunkl embedding. Similarly, using the "radial 
parts map", we will also realize $U_{\chi}$ as the same subalgebra of $\mathfrak{D}\left(\mathfrak{h}_{\mathrm{reg}}\right)$. The Dunkl embedding is the map $\Theta_{\boldsymbol{h}}: H_{\boldsymbol{h}} \rightarrow \mathfrak{D}\left(\mathfrak{h}_{\text {reg }}\right) \rtimes \mathbb{Z}_{m}$ defined by

$$
\Theta_{\boldsymbol{h}}(y)=\frac{\mathrm{d}}{\mathrm{d} x}+\frac{m}{x} \sum_{i=0}^{m-1} h_{i} e_{i}, \quad \Theta_{\boldsymbol{h}}(x)=x \quad \text { and } \quad \Theta_{\boldsymbol{h}}(\varepsilon)=\varepsilon .
$$

The algebra $\mathfrak{D}\left(\mathfrak{h}_{\text {reg }}\right) \rtimes \mathbb{Z}_{m}$ is filtered by order of differential operators, that is, $\operatorname{deg}(\mathrm{d} / \mathrm{d} x)=1$ and $\operatorname{deg}(x)=\operatorname{deg}(\varepsilon)=0$. If we define a filtration on $H_{\boldsymbol{h}}$ by setting $\operatorname{deg}(y)=1$ and $\operatorname{deg}(x)=\operatorname{deg}(\varepsilon)=0$, then the map $\Theta_{\boldsymbol{h}}$ is filter preserving. Localizing $H_{h}$ at the regular element $x$ provides an isomorphism

$$
\Theta_{\boldsymbol{h}}: H_{\boldsymbol{h}}\left[x^{-1}\right] \stackrel{\sim}{\rightarrow} \mathfrak{D}\left(\mathfrak{h}_{\text {reg }}\right) \rtimes \mathbb{Z}_{m} .
$$

Therefore $\Theta_{\boldsymbol{h}}$ is injective. Applying the trivial idempotent produces

$$
\Theta_{\boldsymbol{h}}: e H_{\boldsymbol{h}} e \longrightarrow e \mathfrak{D}\left(\mathfrak{h}_{\mathrm{reg}}\right) e \simeq \mathfrak{D}\left(\mathfrak{h}_{\mathrm{reg}}\right)^{\mathbb{Z}_{m}}
$$

Let us note that $\operatorname{gr}\left(H_{\boldsymbol{h}}\right) \simeq \mathbb{C}[x, y] \rtimes \mathbb{Z}_{m}$ and $\operatorname{gr}\left(e H_{\boldsymbol{h}} e\right) \simeq \mathbb{C}[x, y]^{\mathbb{Z}_{m}}$. Therefore $e H_{\boldsymbol{h}} e$ is generated by $x^{m} e$, xye and $y^{m} e$. Since $\Theta_{\boldsymbol{h}}\left(y e_{i}\right)=\left(\mathrm{d} / \mathrm{d} x+(m / x) h_{i}\right) e_{i}$ we get

$$
\Theta_{\boldsymbol{h}}\left(y^{m} e\right)=\prod_{i=1}^{m}\left(\frac{\mathrm{d}}{\mathrm{d} x}+\frac{m}{x} h_{i}\right) \quad \text { and } \quad \Theta_{\boldsymbol{h}}(x y e)=x \frac{\mathrm{d}}{\mathrm{d} x}+m h_{m} .
$$

We note that

$$
\Theta_{\boldsymbol{h}}\left(y^{m} e\right)\left(x^{r}\right)=\prod_{i=1}^{m}\left(r-m+i+m h_{i}\right) x^{r-m} .
$$

6E. The radial parts map. In this subsection we show that $U_{\chi} \simeq \Theta_{\boldsymbol{h}}\left(e H_{\boldsymbol{h}} e\right)$. The isomorphism we describe is not new, it was first constructed by Holland [1999] (see also [Kuwabara 2008]), but we give it in order to fix parameters. There is a natural embedding $\mathfrak{h} \hookrightarrow \operatorname{Rep}(Q, v)$ given by $x \mapsto(x, \ldots, x)$. This defines a surjective morphism $\mathbb{C}[\operatorname{Rep}(Q, v)] \rightarrow \mathbb{C}[\mathfrak{h}], x_{i} \mapsto x$, which descends to a "Chevalley isomorphism"

$$
\rho: \mathbb{C}[\operatorname{Rep}(Q, v)]^{\mathbb{\mathbb { T }}^{m-1}} \stackrel{\sim}{\longrightarrow}[\mathfrak{C}]^{\mathbb{Z}_{m}}, \quad x_{1} \cdots x_{m} \mapsto x^{m} .
$$

Define a section

$$
\rho^{-1}: \mathbb{C}[\mathfrak{h}] \longrightarrow \mathbb{C}[\operatorname{Rep}(Q, v)]\left[x_{i}^{1 / m} \mid i \in[1, m]\right] \quad \text { by } x^{r} \mapsto x_{1}^{r / m} \cdots x_{m}^{r / m} .
$$

This can be extended to a twisted Harish-Chandra morphism

$$
\hat{\mathfrak{R}}_{\boldsymbol{h}}: \mathscr{D}(\operatorname{Rep}(Q, v))^{\mathbb{T}} \longrightarrow \mathfrak{D}\left(\mathfrak{h}_{\mathrm{reg}}\right)^{\mathbb{Z}_{m}}
$$


given by

$\hat{\mathfrak{R}}_{\boldsymbol{h}}(D)(f)=\rho\left(\delta_{\boldsymbol{h}}^{-1} D\left(\rho^{-1}(f) \delta_{\boldsymbol{h}}\right)\right) \quad$ for all $f \in \mathbb{C}[\mathfrak{h}], \quad$ where $\delta_{\boldsymbol{h}}=\prod_{i=1}^{m} x_{i}^{h_{i}+\frac{i-m}{m}}$.

Calculating the action of $\hat{\mathfrak{R}}_{\boldsymbol{h}}\left(\partial_{1} \cdots \partial_{m}\right)$ on $x^{r}$ and comparing with (18) shows that

$$
\hat{\mathfrak{R}}_{\boldsymbol{h}}\left(m^{m} \cdot \partial_{1} \cdots \partial_{m}\right)=\Theta_{\boldsymbol{h}}\left(y^{m} e\right) .
$$

Similarly,

$$
\hat{\mathfrak{R}}_{\boldsymbol{h}}\left(x_{1} \cdots x_{m}\right)=\Theta_{\boldsymbol{h}}\left(x^{m} e\right) \quad \text { and } \quad \hat{\mathfrak{R}}_{\boldsymbol{h}}\left(x_{i} \partial_{i}\right)=\frac{1}{m}\left(x \frac{\mathrm{d}}{\mathrm{d} x}+m h_{i}+i-m\right) .
$$

This implies that $\hat{\mathfrak{R}}_{\boldsymbol{h}}$ defines a surjection $\mathfrak{D}(\operatorname{Rep}(Q, v))^{\mathbb{\pi}^{m-1}} \rightarrow \Theta_{\boldsymbol{h}}\left(e H_{\boldsymbol{h}} e\right)$. We fix

$$
\chi_{i}=h_{i}-h_{m}+\frac{i-m}{m}, \quad i \in[1, m-1] .
$$

Then $\hat{\mathfrak{R}}_{\boldsymbol{h}}\left(x_{i} \partial_{i}-x_{m} \partial_{m}-\chi_{i}\right)=0$ and $\hat{\mathfrak{R}}_{\boldsymbol{h}}$ descends to a surjective morphism

$$
\mathfrak{R}_{\boldsymbol{h}}: \mathrm{U}_{\chi} \longrightarrow \Theta_{\boldsymbol{h}}\left(e H_{h} e\right) .
$$

As above, $\mathfrak{D}(\operatorname{Rep}(Q, v))$ is a filtered algebra by setting $\operatorname{deg}\left(\partial_{i}\right)=1$ and $\operatorname{deg}\left(x_{i}\right)=0$ for $i \in[1, m]$. This induces a filtration on $\mathrm{U}_{\chi}$ and we see from the definitions that $\mathfrak{R}_{h}$ is filter preserving. Therefore we get a morphism of associated graded algebras

$$
\operatorname{gr} \Re_{h}: \operatorname{gr}\left(U_{\chi}\right) \longrightarrow \operatorname{gr}\left(e H_{h} e\right) .
$$

Now [Holland 1999, Proposition 2.4] says that

$$
\operatorname{gr}\left(\mathrm{U}_{\chi}\right)=\mathbb{C}\left[\mu^{-1}(0)\right]^{\mathbb{\mathbb { T }}^{m-1}} \simeq \mathbb{C}[x, y]^{\mathbb{Z}_{m}}=\operatorname{gr}\left(e H_{\boldsymbol{h}} e\right) .
$$

This isomorphism is realized by $x_{1} \cdots x_{m} \mapsto x^{m}, m^{m} \cdot y_{1} \cdots y_{m} \mapsto y^{m}$ and $x_{1} y_{1} \mapsto$ $(1 / m) \cdot x y$. But we see from above that this is precisely what $\mathrm{gr} \mathfrak{R}_{\boldsymbol{h}}$ does to the principal symbols of the generators $m^{m} \cdot \partial_{1} \cdots \partial_{m}, x_{1} \cdots x_{m}$ and $x_{1} \partial_{1}$ of $\mathrm{U}_{\chi}$. Therefore $\mathrm{gr} \mathfrak{R}_{\boldsymbol{h}}$ is an isomorphism and hence $\Theta_{\boldsymbol{h}}^{-1} \circ \mathfrak{R}_{\boldsymbol{h}}: \mathrm{U}_{\chi} \stackrel{\sim}{\rightarrow} e H_{\boldsymbol{h}} e$ is a filtrationpreserving isomorphism.

6F. Localization of $\boldsymbol{H}_{\boldsymbol{h}}\left(\mathbb{Z}_{\boldsymbol{m}}\right)$. As noted in Proposition 6.2, the hypertoric varieties $Y(A, \delta)$ are all isomorphic provided $\delta$ does not belong to a wall in $\mathbb{X}_{\mathbb{Q}}$. Therefore, for any $\chi \in \mathfrak{t}^{*}$, we may refer to the sheaf $\mathscr{A}_{\chi}$ on the minimal resolution $\left(\mathbb{C}^{2} / \mathbb{Z}_{m}\right)^{\sim}$, but the reader should be aware that in doing so we have implicitly fixed an identification $\left(\mathbb{C}^{2} / \mathbb{Z}_{m}\right)^{\sim}=Y(A, \delta)$. Recall the union of hyperplanes $\mathscr{b} \subset \mathbb{C}^{m}$ defined in Lemma 6.1. 
Theorem 6.3. Choose $\boldsymbol{h} \in \mathbb{C}^{m} \backslash \mathscr{C}$ and let $\chi$ be defined by (19). Write $\mathscr{A}_{\boldsymbol{h}}:=\mathscr{A}_{\chi}$ for the sheaf of $W$-algebras on $\left(\mathbb{C}^{2} / \mathbb{Z}_{m}\right)^{\sim}$. Then the functor

$$
\operatorname{Hom}_{\operatorname{Mod}_{F}^{\mathrm{good}}\left(\mathscr{A}_{\boldsymbol{h}}\right)}\left(\mathscr{A}_{\boldsymbol{h}}, \cdot\right)
$$

defines an equivalence of categories $\operatorname{Mod}_{F}^{\text {good }}\left(\mathscr{A}_{\boldsymbol{h}}\right) \stackrel{\sim}{\longrightarrow} e H_{h} e-\bmod$ with quasiinverse $A_{h} \otimes_{e H_{h} e}(\cdot)$. Moreover, the functor

$$
H_{h} e \otimes_{e H_{h} e} \operatorname{Hom}_{\operatorname{Mod}_{F}^{\mathrm{good}}\left(\mathscr{A}_{h}\right)}\left(\mathscr{A}_{\boldsymbol{h}}, \cdot\right)
$$

defines an equivalence of categories $\operatorname{Mod}_{F}^{\text {good }}\left(\mathscr{A}_{\boldsymbol{h}}\right) \stackrel{\sim}{\longrightarrow} H_{\boldsymbol{h}}$-mod with quasiinverse $A_{h} \otimes_{e H_{h} e} e H_{h} \otimes_{H_{h}}(\cdot)$.

Proof. The condition $\chi_{i} \neq \chi_{j}$ for $i \neq j \in[1, m-1]$ translates, via (19), into $h_{i}-h_{j}+(i-j) / m \neq 0$ for all $i \neq j \in[1, m-1]$. Similarly, the condition $\chi_{i} \neq 0$ for all $i \in[1, m-1]$ translates into $h_{i}-h_{m}+\frac{i-m}{m} \neq 0$ for all $i \in[1, m-1]$. Therefore the linear map $\mathbb{C}^{m} \rightarrow \mathbb{C}^{m-1}$ defined by (19) maps the union of hyperplanes $\mathscr{C}$ onto

$$
\left.\left\{\chi \in \mathbb{C}^{m-1} \mid \chi_{i}=\chi_{j} \text { for } i \neq j \in[1, m-1] \text { or } \chi_{i}=0 \text { for } i \in[1, m-1]\right)\right\},
$$

which is precisely the union of the G.I.T. walls in $\mathbb{C}^{m-1}$. Therefore Lemma 6.1 implies that $U_{\chi}$ has finite global dimension when $\chi$ lies in the interior of some G.I.T. cone $C$. Now the theorem follows from Corollary 5.3.

Remark 6.4. In the above situation it is possible to explicitly calculate the sets $2_{\chi}$ and hence describe the partial ordering on comparability classes as defined in Section 5A. However the answer is not very illuminating.

Finally, we would just like to note the various forms in which the rational Cherednik algebra $H_{\boldsymbol{h}}\left(\mathbb{Z}_{m}\right)$ appears in the literature. It is isomorphic to the deformed preprojective algebra of type $A$ as studied in [Crawley-Boevey and Holland 1998]. It is well-known that its spherical subalgebra $e H_{\boldsymbol{h}}\left(\mathbb{Z}_{m}\right) e$ coincides with a "generalized $U\left(\mathfrak{s l}_{2}\right)$-algebra", as studied by Hodges [1993] and Smith [1990]. Combining this fact with Premet's results [2002] shows that $e H_{h}\left(\mathbb{Z}_{m}\right) e$ is also isomorphic to the finite $W$-algebra associated to $\mathfrak{g l}_{m}(\mathbb{C})$ at a subregular nilpotent element. Recently, Losev [2012] has constructed explicit isomorphisms between the spherical subalgebra of certain rational Cherednik algebras and their related finite $\mathcal{W}$-algebras, which as a special case gives the above mentioned isomorphism.

Musson [2005] and Boyarchenko [2007] have studied a certain localization of $e H_{\boldsymbol{h}}\left(\mathbb{Z}_{m}\right) e$ by using the formalism of directed algebras (or $\mathbb{Z}$-algebras). Analogous localizations for finite $\mathcal{W}$-algebras were established by Ginzburg [2009]. Recently, Dodd and Kremnizer [2009] described a localization theorem for finite $\mathcal{W}$-algebras 
in the spirit of Kashiwara-Rouquier, and in particular for the finite $W$-algebra isomorphic to $e H_{\boldsymbol{h}}\left(\mathbb{Z}_{m}\right) e$. However, their result is via a different quantum Hamiltonian reduction than the one used in Theorem 6.3.

In [Kuwabara 2010], the second author gives an explicit description of the standard modules $\Delta\left(e_{i}\right)$ and simple modules $L\left(e_{i}\right)$ as sheaves of $\mathscr{A}_{\boldsymbol{h}}$-modules on the minimal resolution.

\section{Acknowledgments}

The authors would like to express their gratitude to Professors Iain Gordon, Masaki Kashiwara and Raphael Rouquier for their helpful comments and many fruitful discussions. They would also like to thank Professors Bernd Sturmfels and Tamás Hausel for outlining the proof of Lemma 4.7. The first author thanks Maurizio Martino and Olaf Schnürer for stimulating discussions.

\section{References}

[Beǔlinson and Bernstein 1981] A. Běllinson and J. Bernstein, "Localisation de $g$-modules", C. R. Acad. Sci. Paris Sér. I Math. 292:1 (1981), 15-18. MR 82k:14015 Zbl 0476.14019

[Van den Bergh 1991] M. Van den Bergh, "Differential operators on semi-invariants for tori and weighted projective spaces", pp. 255-272 in Topics in invariant theory (Paris, 1989/1990), edited by M.-P. Malliavin-Brameret, Lecture Notes in Math. 1478, Springer, Berlin, 1991. MR 93h:16046 Zbl 0802.13005

[Bielawski and Dancer 2000] R. Bielawski and A. S. Dancer, "The geometry and topology of toric hyperkähler manifolds”, Comm. Anal. Geom. 8:4 (2000), 727-760. MR 2002c:53078 Zbl 0992.53034

[Björk 1979] J.-E. Björk, Rings of differential operators, North-Holland Mathematical Library 21 , North-Holland Publishing Co., Amsterdam, 1979. MR 82g:32013 Zbl 0499.13009

[Boyarchenko 2007] M. Boyarchenko, "Quantization of minimal resolutions of Kleinian singularities”, Adv. Math. 211:1 (2007), 244-265. MR 2008f:14001 Zbl 1138.16012

[Bruns and Herzog 1993] W. Bruns and J. Herzog, Cohen-Macaulay rings, Cambridge Studies in Advanced Mathematics 39, Cambridge University Press, 1993. MR 95h:13020 Zbl 0788.13005

[Cassens and Slodowy 1998] H. Cassens and P. Slodowy, "On Kleinian singularities and quivers", pp. 263-288 in Singularities (Oberwolfach, 1996), edited by V. I. Arnold et al., Progr. Math. 162, Birkhäuser, Basel, 1998. MR 99k:14003 Zbl 0957.14004

[Cox et al. 2007] D. Cox, J. Little, and D. O'Shea, Ideals, varieties, and algorithms: An introduction to computational algebraic geometry and commutative algebra, 3rd ed., Undergraduate Texts in Mathematics, Springer, New York, 2007. MR 2007h:13036 Zbl 1118.13001

[Crawley-Boevey and Holland 1998] W. Crawley-Boevey and M. P. Holland, "Noncommutative deformations of Kleinian singularities", Duke Math. J. 92:3 (1998), 605-635. MR 99f:14003 Zbl 0974.16007

[Dodd and Kremnizer 2009] C. Dodd and K. Kremnizer, "A localization theorem for finite Walgebras", preprint, 2009. arXiv 0911.2210v1

[Fu 2006] B. Fu, "A survey on symplectic singularities and symplectic resolutions", Ann. Math. Blaise Pascal 13:2 (2006), 209-236. MR 2007j:14019 Zbl 1116.14008 
[Ginzburg 2003] V. Ginzburg, “On primitive ideals”, Selecta Math. (N.S.) 9:3 (2003), 379-407. MR 2005f:16039 Zbl 1041.16016

[Ginzburg 2009] V. Ginzburg, "Harish-Chandra bimodules for quantized Slodowy slices", Represent. Theory 13 (2009), 236-271. MR 2010g:17009 Zbl 05706397

[Ginzburg et al. 2009] V. Ginzburg, I. Gordon, and J. T. Stafford, "Differential operators and Cherednik algebras”, Selecta Math. (N.S.) 14:3-4 (2009), 629-666. MR 2011b:16089 Zbl 1193.14007

[Hartshorne 1977] R. Hartshorne, Algebraic geometry, Graduate Texts in Mathematics 52, Springer, New York, 1977. MR 57 \#3116 Zbl 0367.14001

[Hausel and Sturmfels 2002] T. Hausel and B. Sturmfels, “Toric hyperKähler varieties”, Doc. Math. 7 (2002), 495-534. MR 2004i:53054 Zbl 1029.53054

[Hodges 1993] T. J. Hodges, "Noncommutative deformations of type- $A$ Kleinian singularities", $J$. Algebra 161:2 (1993), 271-290. MR 94i:14038 Zbl 0807.16029

[Holland 1999] M. P. Holland, "Quantization of the Marsden-Weinstein reduction for extended Dynkin quivers”, Ann. Sci. École Norm. Sup. (4) 32:6 (1999), 813-834. MR 2001a:16042 Zbl 1036. 16024

[Hotta et al. 2008] R. Hotta, K. Takeuchi, and T. Tanisaki, D-modules, perverse sheaves, and representation theory, Progress in Mathematics 236, Birkhäuser, Boston, MA, 2008. MR 2008k:32022 Zbl 1136.14009

[Kashiwara and Rouquier 2008] M. Kashiwara and R. Rouquier, "Microlocalization of rational Cherednik algebras", Duke Math. J. 144:3 (2008), 525-573. MR 2010a:16053 Zbl 1147.14002

[Kempf 1978] G. R. Kempf, "Instability in invariant theory", Ann. of Math. (2) 108:2 (1978), 299-316. MR 80c:20057 Zbl 0406.14031

[Kontsevich 2001] M. Kontsevich, "Deformation quantization of algebraic varieties", Lett. Math. Phys. 56:3 (2001), 271-294. MR 2002j:53117 Zbl 1081.14500

[Kraft 1984] H. Kraft, Geometrische Methoden in der Invariantentheorie, Aspects of Mathematics D1, Vieweg, Braunschweig, 1984. MR 86j:14006 Zbl 0569.14003

[Kronheimer 1989] P. B. Kronheimer, "The construction of ALE spaces as hyper-Kähler quotients", J. Differential Geom. 29:3 (1989), 665-683. MR 90d:53055 Zbl 0671.53045

[Kuwabara 2008] T. Kuwabara, "Characteristic cycles of standard modules for the rational Cherednik algebra of type $\mathbb{Z} / l \mathbb{Z}$ ”, J. Math. Kyoto Univ. 48:1 (2008), 167-217. MR 2009k:16055 Zbl 1190.16042

[Kuwabara 2010] T. Kuwabara, "Representation theory of rational cherednik algebras of type $\mathbb{Z} / l \mathbb{Z}$ via microlocal analysis", preprint, 2010. To appear in Publ. Res. Int. Math. Sci. arXiv 1003.3407

[Losev 2012] I. Losev, "Isomorphisms of quantizations via quantization of resolutions", Adv. Math. 231:3-4 (2012), 1216-1270. MR 2964603

[McConnell and Robson 2001] J. C. McConnell and J. C. Robson, Noncommutative Noetherian rings, Revised ed., Graduate Studies in Mathematics 30, American Mathematical Society, Providence, RI, 2001. MR 2001i:16039 Zbl 0980.16019

[Musson 2005] I. M. Musson, "Hilbert schemes and noncommutative deformations of type A Kleinian singularities", J. Algebra 293:1 (2005), 102-129. MR 2006g:16066 Zbl 1082.14008

[Musson and Van den Bergh 1998] I. M. Musson and M. Van den Bergh, "Invariants under tori of rings of differential operators and related topics", Mem. Amer. Math. Soc. 136:650 (1998), viii+85. MR 99i:16051 Zbl 0928.16019

[Namikawa 2001] Y. Namikawa, "Extension of 2-forms and symplectic varieties", J. Reine Angew. Math. 539 (2001), 123-147. MR 2002i:32011 Zbl 0996.53050 
[Oda and Park 1991] T. Oda and H. S. Park, "Linear Gale transforms and Gel'fand-KapranovZelevinskij decompositions”, Tohoku Math. J. (2) 43:3 (1991), 375-399. MR 92d:14042 Zbl 0782 52006

[Polesello and Schapira 2004] P. Polesello and P. Schapira, "Stacks of quantization-deformation modules on complex symplectic manifolds", Int. Math. Res. Not. 2004:49 (2004), 2637-2664. MR 2005e:32018 Zbl 1086.53107

[Premet 2002] A. Premet, "Special transverse slices and their enveloping algebras", Adv. Math. 170:1 (2002), 1-55. MR 2003k:17014 Zbl 1005.17007

[Proudfoot 2008] N. J. Proudfoot, "A survey of hypertoric geometry and topology", pp. 323-338 in Toric topology, edited by M. Harada et al., Contemp. Math. 460, Amer. Math. Soc., Providence, RI, 2008. MR 2010a:14087 Zbl 1151.53042

[Proudfoot and Webster 2007] N. Proudfoot and B. Webster, "Intersection cohomology of hypertoric varieties", J. Algebraic Geom. 16:1 (2007), 39-63. MR 2008m:14037 Zbl 1119.14016

[Rouquier 2008] R. Rouquier, " $q$-Schur algebras and complex reflection groups”, Mosc. Math. J. 8:1 (2008), 119-158, 184. MR 2010b:20081 Zbl 1213.20007

[Smith 1990] S. P. Smith, "A class of algebras similar to the enveloping algebra of sl(2)", Trans. Amer. Math. Soc. 322:1 (1990), 285-314. MR 91b:17013 Zbl 0732.16019

[Weibel 1994] C. A. Weibel, An introduction to homological algebra, Cambridge Studies in Advanced Mathematics 38, Cambridge University Press, 1994. MR 95f:18001 Zbl 0797.18001

Received July 22, 2011.

GWYN BELLAMY

SCHOOL OF MATHEMATICS AND STATISTICS

UNIVERSITY OF GLASGOW

UNIVERSITY GARDENS

GLASGOW

G12 8QW

UNITED KINGDOM

gwyn.bellamy@glasgow.ac.uk

TOSHIRO KUWABARA

DEPARTMENT OF MATHEMATICS

NATIONAL RESEARCH UNIVERSity Higher SCHOOL OF ECONOMICS

7 VAVILOVA STREET

Moscow

117312

RUSSIA

toshiro.kuwa@gmail.com 


\title{
PACIFIC JOURNAL OF MATHEMATICS
}

\author{
http://pacificmath.org
}

Founded in 1951 by E. F. Beckenbach (1906-1982) and F. Wolf (1904-1989)

\section{EDITORS}

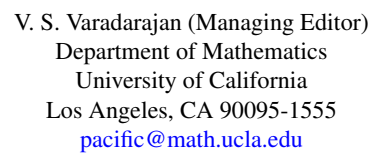

Don Blasius

Department of Mathematics University of California

Los Angeles, CA 90095-1555

blasius@math.ucla.edu

Robert Finn

Department of Mathematics

Stanford University

Stanford, CA 94305-2125

finn@math.stanford.edu

Alexander Merkurjev

Department of Mathematics

University of California

Los Angeles, CA 90095-1555

merkurev@math.ucla.edu

\author{
Vyjayanthi Chari \\ Department of Mathematics \\ University of California \\ Riverside, CA 92521-0135 \\ chari@math.ucr.edu \\ Kefeng Liu \\ Department of Mathematics \\ University of California \\ Los Angeles, CA 90095-1555 \\ liu@math.ucla.edu \\ Sorin Popa \\ Department of Mathematics \\ University of California \\ Los Angeles, CA 90095-1555 \\ popa@math.ucla.edu \\ Paul Yang \\ Department of Mathematics \\ Princeton University \\ Princeton NJ 08544-1000 \\ yang@math.princeton.edu
}

\section{PRODUCTION}

Silvio Levy, Scientific Editor, pacific@math.berkeley.edu

\section{SUPPORTING INSTITUTIONS}

ACADEMIA SINICA, TAIPEI

CALIFORNIA INST. OF TECHNOLOGY

INST. DE MATEMÁTICA PURA E APLICADA

KEIO UNIVERSITY

MATH. SCIENCES RESEARCH INSTITUTE

NEW MEXICO STATE UNIV.

OREGON STATE UNIV.

\author{
STANFORD UNIVERSITY \\ UNIV. OF BRITISH COLUMBIA \\ UNIV. OF CALIFORNIA, BERKELEY \\ UNIV. OF CALIFORNIA, DAVIS \\ UNIV. OF CALIFORNIA, LOS ANGELES \\ UNIV. OF CALIFORNIA, RIVERSIDE \\ UNIV. OF CALIFORNIA, SAN DIEGO \\ UNIV. OF CALIF., SANTA BARBARA
}

\author{
Daryl Cooper \\ Department of Mathematics \\ University of California \\ Santa Barbara, CA 93106-3080 \\ cooper@math.ucsb.edu \\ Jiang-Hua Lu \\ Department of Mathematics \\ The University of Hong Kong \\ Pokfulam Rd., Hong Kong \\ jhlu@maths.hku.hk
}

\section{Jie Qing}

Department of Mathematics

University of California

Santa Cruz, CA 95064

qing@cats.ucsc.edu

These supporting institutions contribute to the cost of publication of this Journal, but they are not owners or publishers and have no responsibility for its contents or policies.

See inside back cover or pacificmath.org for submission instructions.

The subscription price for 2012 is US \$420/year for the electronic version, and \$485/year for print and electronic.

Subscriptions, requests for back issues from the last three years and changes of subscribers address should be sent to Pacific Journal of Mathematics, P.O. Box 4163, Berkeley, CA 94704-0163, U.S.A. Prior back issues are obtainable from Periodicals Service Company, 11 Main Street, Germantown, NY 12526-5635. The Pacific Journal of Mathematics is indexed by Mathematical Reviews, Zentralblatt MATH, PASCAL CNRS Index, Referativnyi Zhurnal, Current Mathematical Publications and the Science Citation Index.

The Pacific Journal of Mathematics (ISSN 0030-8730) at the University of California, c/o Department of Mathematics, 969 Evans Hall, Berkeley, CA 94720-3840, is published monthly except July and August. Periodical rate postage paid at Berkeley, CA 94704, and additional mailing offices. POSTMASTER: send address changes to Pacific Journal of Mathematics, P.O. Box 4163, Berkeley, CA 94704-0163.

PJM peer review and production are managed by EditFlow ${ }^{\circledR}$ from Mathematical Sciences Publishers.

\section{PUBLISHED BY}

\section{mathematical sciences publishers}

http://msp.org/

A NON-PROFIT CORPORATION

Typeset in LATEX 


\section{PACIFIC JOURNAL OF MATHEMATICS}

Volume $260 \quad$ No. $1 \quad$ November 2012

The decomposition of global conformal invariants: Some technical proofs II

SPYROS ALEXAKIS

On deformation quantizations of hypertoric varieties

GWYN BELLAMY and TOSHIRO KUWABARA

Almost factoriality of integral domains and Krull-like domains

GYU Whan CHANG, HWANKOO KIM and JUNG WOOK LIM

Singularities of free group character varieties

CARLOS FLORENTINO and SEAN LAWTON

Energy identity for the maps from a surface with tension field bounded 181 in $L^{p}$

LI JIAYU and ZHU XIANGRONG

Remarks on some isoperimetric properties of the $k-1$ flow

YU-CHU LIN and DONG-Ho TSAI

Demystifying a divisibility property of the Kostant partition function 215

KAROLA MÉSZÁros

Exceptional Lie algebras, SU(3), and Jordan pairs

PIERO TRUINI

Lower estimate of Milnor number and characterization of isolated homogeneous hypersurface singularities

Stephen S.-T. Yau and HuAiqing Zuo 